\title{
On the Spectrum of Hilbert Matrix Operator
}

\author{
Bernd Silbermann®
}

\begin{abstract}
The Hilbert matrix

$$
\mathcal{H}_{\lambda}=\left(\frac{1}{n+m+\lambda}\right)_{n, m=0}^{\infty}, \quad \lambda \neq 0,-1,-2, \ldots
$$

generates a bounded linear operator in the Hardy spaces $H^{p}$ and in the $l^{p}$-spaces. The aim of this paper is to study the spectrum of this operator in the spaces mentioned. In a sense, the presented investigation continues earlier works of various authors. More information concerning the history of the topic can be found in the introduction.
\end{abstract}

Mathematics Subject Classification. Primary: 47B35, 47B38; Secondary: 47B33, 45E10.

Keywords. Toeplitz and Hankel operators, Hilbert matrix operator, Spectrum, Essential and point spectrum, Eigenfunctions.

\section{Introduction}

The classical Hilbert matrix

$$
\mathcal{H}_{1}=\left(\frac{1}{n+m+1}\right)_{n, m=0}^{\infty}
$$

was introduced by Hilbert in 1894 while working on a problem in theory of orthogonal polynomials. In particular, he studied finite sections of the matrix $\mathcal{H}_{1}$ and observed that they are strongly ill-conditioned. This fact plays an important role in numerical analysis. In this work however, we are merely interested in the matrix $\mathcal{H}_{1}$ itself and in its generalization

$$
\mathcal{H}_{\lambda}=\left(\frac{1}{n+m+\lambda}\right)_{n, m=0}^{\infty}, \quad \lambda \neq 0,-1,-2, \ldots
$$

thought as linear operators acting on the spaces $l^{p}$ of $p$-summable sequences and on Hardy spaces $H^{p}$. In what follows, unless specified otherwise, we assume that $1<p<\infty$. 
Let us recall the properties of the operator $\mathcal{H}_{\lambda}$. We denote by $\mathbb{Z}$ the set of all integers, by $\mathbb{Z}_{+}$the set of non-negative integers, by $\mathbb{Z}_{-}$the set of non-positive integers and by $\mathbb{D}$ the open unit disc in the complex plane $\mathbb{C}$, i.e.

$$
\mathbb{D}:=\{z \in \mathbb{C}:|z|<1\} .
$$

Theorem 1.1. (Hilbert's Inequality). If the sequence $\left(a_{n}\right)_{n \in \mathbb{Z}_{+}}$belongs to $l^{p}$, then

$$
\left(\sum_{n=0}^{\infty}\left|\frac{a_{k}}{m+k+1}\right|^{p}\right)^{1 / p} \leq \frac{\pi}{\sin (\pi / p)}\left(\sum_{n=0}^{\infty}\left|a_{k}\right|^{p}\right)^{1 / p}
$$

and $\pi /(\sin (\pi / p))$ is the best possible constant in the inequality (1.3).

Thus the operator $\mathcal{H}_{1}$ is bounded on the space $l^{p}$. On the other hand, a lot of effort has been spent on studying Hilbert matrix operator $\mathcal{H}_{\lambda}$ acting in spaces constituted by subspaces of the space $\mathcal{A}(\mathbb{D})$ of all holomorphic functions on $\mathbb{D}$. The idea is to let the matrix $\mathcal{H}_{\lambda}$ act on the Taylor coefficient sequences of functions from the corresponding subspace. The so-defined operator is also denoted by $\mathcal{H}_{\lambda}$ and the reader can consult [23] for more information concerning this matter.

In the period from 1950 to 1960 the spectral properties of the operator $\mathcal{H}_{1}$ and its generalization $\mathcal{H}_{\lambda}$ have been vigorously studied. In particular, remarkable results are obtained for the spectrum of $\mathcal{H}_{1}$ in $l^{2}$ and its latent roots.

Definition 1.2. A latent root for $\mathcal{H}_{\lambda}, \lambda \neq \mathbb{Z}_{-}$is a complex number $\alpha$ such that there is a non-zero sequence $\left(x_{n}\right)_{n=0}^{\infty}, x_{n} \in \mathbb{C}$ with the property

$$
\sum_{m=0}^{\infty} \frac{x_{m}}{n+m+\lambda}=\alpha x_{n}, \quad n=0,1, \ldots .
$$

We note that it is not supposed the operator $\mathcal{H}_{\lambda}$ acts on a Banach space.

Latent roots of $\mathcal{H}_{\lambda}$ also attracted considerable attentions and the most general results are obtained by Rosenblum [18] and Hill [9]. For instance, the following remarkable result is true.

Theorem 1.3. (cf. Hill [9]). Let $\mu$ be a complex number such that $0<$ Re $\mu \leq$ $1 / 2$ and $x_{n}=x_{n}(\lambda, \mu)$ be the sequence defined by

$$
x_{n}:=\sum_{k=0}^{n}\left(\begin{array}{l}
n \\
k
\end{array}\right) \frac{(-1)^{-n} \Gamma(k+\mu) \Gamma(k+1-\mu)}{\Gamma(k+\lambda) \Gamma(k+1)}, \quad n=0,1, \ldots .
$$

Then

$$
\sum_{m=0}^{\infty} \frac{x_{m}}{n+m+\lambda}=\frac{\pi}{\sin (\pi \mu)} x_{n}, \quad n=0,1, \ldots,
$$

i.e. $\pi /(\sin (\pi \mu))$ is a latent root of $\mathcal{H}_{\lambda}$.

Some other prominent results to be mentioned here are related to the spectrum of Hilbert matrix operators. 
Theorem 1.4. (cf. Magnus [16]). The spectrum of $\mathcal{H}_{1}$ on $l^{2}$ is the interval $[0, \pi]$, and there are no eigenvalues.

On the other hand, taking into account that for real $\lambda$ the operator $\mathcal{H}_{\lambda}: l^{2} \rightarrow l^{2}$ is self-adjoint, Rosenblum explicitly diagonalized $\mathcal{H}_{\lambda}$ and described its spectrum.

Theorem 1.5. (cf. Rosenblum [19]). Let $\lambda=1-k$, where $k$ is a real number such that $k \notin \mathbb{N}$. Then the following assertions hold:

(i) For any real $\lambda$ the operator $\mathcal{H}_{\lambda}: l^{2} \rightarrow l^{2}$ has a continuous spectrum of multiplicity one located on the interval $[0, \pi]$.

(ii) If $k \leq 1 / 2$, then the operator $\mathcal{H}_{\lambda}$ has no point spectrum.

(iii) If $k>1 / 2$ and $u$ and $v$ are, respectively, the largest non-negative integers such that

$$
2 u<k-\frac{1}{2} \quad \text { and } \quad 2 v<k-\frac{3}{2},
$$

then $\pi / \sin (\pi k)$ and $-\pi / \sin (\pi k)$ are the only eigenvalues of the operator $\mathcal{H}_{\lambda}$ and they have multiplicity $u+1$ and $v+1$, respectively.

Another important work is due to Aleman et al. [1] who, in particular, described the point spectrum of the operator $\mathcal{H}_{\lambda}$ considered on Hardy spaces $H^{p}$. Besides, Gonsales [6] draw the attention to the spectrum description for the Hilbert matrix operator acting on spaces $l^{p}$ and to the essential spectrum of $\mathcal{H}_{\lambda}$. The main tool to achieve this aim is the theory of Toeplitz and Hankel operators with piecewise continuous generating functions. Therefore, in Sect. 2, we recall some features of these operators acting on $H^{p}$ or $l^{p}$ spaces. Section 3 contains the description of the essential spectrum of $\mathcal{H}_{\lambda}$ in the spaces $H^{p}$ and $l^{p}, 1<p<\infty$. Section 4 deals with the point spectrum of $\mathcal{H}_{\lambda}$ with respect to $H^{p}, 1<p<\infty$ and $\lambda \in \mathbb{C} \backslash \mathbb{Z}$. If $\lambda \in \mathbb{N}$, the spectrum of $\mathcal{H}_{\lambda}$ with respect to $H^{p}$ is studied in Sect. 5. The results of Sects. 3-5 are summarized in the Main Theorem formulated in Sect. 6. Section 7 is devoted to the description of the spectrum of $\mathcal{H}_{\lambda}$ acting in $l^{p}$-spaces. It is shown that this description follows from the Main Theorem formulated in Sect. 6. Section 8 provides an outlook for extension of the present work results.

\section{Spaces and Operators}

For every Banach space $X$, let $\mathcal{L}(X)$ stand for the Banach algebra of all bounded linear operators acting on $X$. We denote by $\mathcal{K}(X)$ the closed twosided ideal of all compact operators in $\mathcal{L}(X)$. The quotient algebra $\mathcal{L}(X) / \mathcal{K}(X)$ is called Calkin algebra. For us its importance lies in the fact that the invertibility of the coset $A+\mathcal{K}(X), A \in \mathcal{L}(X)$, in the Calkin algebra is equivalent to the Fredholmness of $A$, i.e. to the finite-dimensionality of the spaces $\operatorname{ker} A:=\{x \in X: A x=0\}$ and coker $A:=X / \operatorname{im} A$. The number

$$
\text { ind } A:=\operatorname{dim} \operatorname{ker} A-\operatorname{dim} \operatorname{coker} A
$$

is called the index of the Fredholm operator $A$. It is also well-known that the condition $\operatorname{dim}$ coker $A<\infty$ is equivalent to the fact that $\operatorname{im} A$ is closed and 
the dimension of the kernel of the adjoint operator $A^{*}$ is finite. Moreover, if $A$ is Fredholm, then $\operatorname{dim} \operatorname{coker} A=\operatorname{dim} \operatorname{ker} A^{*}$.

The following lemma is often used in our considerations.

Lemma 2.1. Let $X_{1}$ and $X_{2}$ be Banach spaces such that $X_{1}$ is continuously and densely embedded into $X_{2}$. Suppose that a given Fredholm operator $A_{1}: X_{1} \rightarrow$ $X_{1}$ can be boundedly extended to a Fredholm operator $A_{2}: X_{2} \rightarrow X_{2}$. If ind $A_{1}=$ ind $A_{2}$, then $\operatorname{ker} A_{1}=\operatorname{ker} A_{2}$.

\subsection{The Spaces $L^{p}$ and $H^{p}$}

Let $\mathbb{T}:=\{z \in \mathbb{C}:|z|=1\}$ be the unit circle in the complex plane. We denote by $L^{p}, 1 \leq p<\infty$ the Banach space of all classes of complex-valued measurable functions on $\mathbb{T}$ summable in the $p$-th power with respect to the Lebesgue measure on $\mathbb{T}$. Further, let $C=C(\mathbb{T})$ and $L^{\infty}=L^{\infty}(\mathbb{T})$ refer to the $C^{*}$-algebras of all continuous functions and all classes of measurable and bounded functions on $\mathbb{T}$, respectively. The norm in $L^{p}, 1 \leq p \leq \infty$ is denoted by $\|\cdot\|_{p}$. It is clear that $C$ is a $C^{*}$-subalgebra of $L^{\infty}$. If we define functions $\chi_{n}(t), n \in \mathbb{Z}$ by $\chi_{n}(t)=t^{n}, t \in \mathbb{T}$, then $\left\{\chi_{n}(t)\right\}_{n \in \mathbb{Z}}$ becomes a Schauder basis for any space $L^{p}, 1<p<\infty$.

Besides, we say that $f \in \mathcal{A}(\mathbb{D})$ belongs to the Hardy space $H^{p}, 1 \leq p \leq$ $\infty$ if

$$
\begin{aligned}
& \|f\|_{H^{p}}:=\sup _{r \in(0,1)}\left(\int_{0}^{2 \pi}\left|f\left(r e^{i \theta}\right)\right|^{p} \frac{d \theta}{2 \pi}\right)^{1 / p}<\infty, \quad p \neq \infty \\
& \|f\|_{H^{\infty}}:=\sup _{z \in \mathbb{D}}|z|<\infty, \quad p=\infty .
\end{aligned}
$$

The so-defined spaces $H^{p}$ are Banach spaces. Let us recall some of their properties.

1. If $f \in H^{p}$ then the limit

$$
\breve{f}\left(e^{i \theta}\right):=\lim _{r \rightarrow 1-0} f\left(r e^{i \theta}\right),
$$

exists for almost all $\theta \in[0,2 \pi]$ and for $p<\infty$ the resulting function $\check{f}$ is $p$-integrable on $\mathbb{T}$. Moreover,

$$
\|\check{f}\|_{L^{p}}=\|f\|_{H^{p}}, \quad 1 \leq p \leq \infty,
$$

(see, e.g. [12, Chapter IV, Section C] and [6, Chapter II, Section 3].

2. The Eq. (2.1) indicates that $H^{p}, 1 \leq p \leq \infty$ can be identified with the closed subspace of $L^{p}$, which consists of boundary values $\check{f}$ of functions $f \in H^{p}$. It can be proved that this subspace coincides with the subset of $L^{p}$, which contains all functions $f$ whose classical Fourier coefficients $\widehat{f}_{n}$ vanish for all $n<0$. It is also denoted by $H^{p}$ and the representation of functions $f \in H^{p}$ in the form

$$
f(x)=\sum_{n=0}^{\infty} \widehat{f}_{l} t^{l}
$$

plays an important role in what follows.

For $1<p<\infty$, the spaces $H^{p}$ are closely connected with the Riesz 
projection $P$, which acts on the set $\mathcal{P}$ of all Laurent polynomials by

$$
P: \sum_{-k}^{k} f_{l} t^{l}=\sum_{0}^{k} f_{l} t^{l}, \quad t \in \mathbb{T} .
$$

The famous Riesz Theorem states that $P$ extends to a bounded linear operator on the whole space $L^{p}, 1<p<\infty$. Besides, it is also clear that $P^{2}=P$ and $H^{p}$ is the image of $L^{p}$ for the Riesz projection, i.e. $H^{p}=P\left(L^{p}\right)$.

3. Fejer-Riesz inequality: If $f \in H^{p}, 1 \leq p<\infty$, then

$$
\int_{-1}^{1}|f(x)|^{p} d x \leq \frac{1}{2}\|\check{f}\|_{p}^{p} .
$$

We recall that $\|f\|_{H^{p}}=\|\check{f}\|_{p}$.

4. If $f \in H^{p}$, then

$$
|f(z)| \leq \frac{C_{p}\|f\|_{p}}{(1-|z|)^{1 / p}}, \quad z \in \mathbb{D} .
$$

5. Let $\arg z$ denote that argument of $z \in \mathbb{C} \backslash\{0\}$ which is in $(-\pi, \pi]$. For $\beta \in \mathbb{C}$ and $t \in \mathbb{T} \backslash\{1\}$, we define the function $\eta_{\beta}(t)=(1-t)^{\beta}$ by

$$
\eta_{\beta}(t):=\exp \{\beta \ln |1-t|+i \beta \arg (1-t)\} \text {. }
$$

Note that $\eta_{\beta}$ is the limit on $\mathbb{T}$ of that branch of $(1-z)^{\beta}$, which is analytic for $|z|<1$ and takes the value 1 at $z=0$. In particular, for $t \in \mathbb{T} \backslash\{1\}$ we have

$$
\left|\eta_{\beta}(t)\right|=\exp \{\operatorname{Re} \beta \ln |1-t|-\operatorname{Im} \beta \arg (1-t)\}=|1-t|^{\operatorname{Re} \beta} b(t)
$$

with a function $b$ invertible in $L^{\infty}$. It follows that $\eta_{\beta} \in L^{p}$ if and only if

$$
-\frac{1}{p}<\operatorname{Re} \beta
$$

hence $\eta_{\beta} \in H^{p}$ if and only if $\beta$ satisfies the inequality (2.2).

\subsection{Toeplitz and Hankel Operators on $\boldsymbol{H}^{p}$.}

Let $a \in L^{\infty}$. Then the Toeplitz operator $T(a): H^{p} \rightarrow H^{p}$ is defined by $h \mapsto P a h$, where $P$ is the Riesz projection. It is clear that $T(a)$ is bounded. Moreover, the mapping $a \mapsto T(a)$ is one-to-one and $\|T(a)\|=\|a\|_{\infty}$. The function $a$ is called the generating function for $T(a)$.

The definition of Hankel operator is a bit more complicated. Let $Q:=$ $I-P$. The image of $Q$ is a subspace of codimension one of the space

$$
\bar{H}^{p}:=\left\{f \in L^{p}: \widehat{f}_{n}=0 \quad \text { for } \quad n \in \mathbb{N}\right\} .
$$

We also need a flip operator $J: L^{p} \rightarrow L^{p}$ defined by

$$
(J f)(t)=\bar{t} f(\bar{t}), \quad t \in \mathbb{T},
$$

where bar denotes the complex conjugation. This operator $J$ changes the orientation of the unit circle $\mathbb{T}$, which is assumed to be counterclockwise 
oriented throughout this paper. We note that the operators $J, P, Q$ and $a I$, $a \in L^{\infty}$ interact with each other as follows:

$$
J^{2}=I, \quad J P J=Q, \quad J Q J=P, J a J=\widetilde{a} I,
$$

and $\widetilde{a}(t):=a(\bar{t}), t \in \mathbb{T}$.

For $a \in L^{\infty}$, the Hankel operator $H(a): H^{p} \rightarrow H^{p}$ is defined by

$$
H(a) h:=P a J h=P a Q J h .
$$

This operator is bounded and $a$ is also called generating function, but unlike Toeplitz operators, the mapping $a \mapsto H(a)$ is not one-to-one. It is worth noting here that the matrix representations of the operators $T(a)$ and $H(a)$ in the Schauder basis $\left\{t^{n}\right\}_{n \in \mathbb{Z}_{+}}$of $H^{p}$, respectively, are

$$
\left(\widehat{a}_{j-i}\right)_{i, j=0}^{\infty} \text { and }\left(\widehat{a}_{j+i+1}\right)_{i, j=0}^{\infty}
$$

where $\widehat{a}_{k}, k \in \mathbb{Z}$ are the Fourier coefficients of $a$.

A function $a: \mathbb{T} \rightarrow \mathbb{C}$ is called piecewise continuous if it has one-sided limits at any point of $\mathbb{T}$. We will, respectively, write $a(t-0)$ and $a(t+0)$ for the left and right limits of $f$ at $t \in \mathbb{T}$, recalling that the unit circle $\mathbb{T}$ is counter-clockwise oriented. The set of all piecewise continuous functions $f$ on $\mathbb{T}$ is denoted by $P C$. To describe the essential spectrum of $\mathcal{H}_{1}$, one can use a highly non-trivial fact that $\mathcal{H}_{1}$ can be identified with a Hankel operator $H(b)$, which belongs to the smallest closed subalgebra $\mathcal{T}_{p}(P C) \subset \mathcal{L}\left(H^{p}\right)$ containing all Toeplitz operators generated by functions $a \in P C$. More exactly, the matrix Hilbert operator $\mathcal{H}_{1}$ on $H^{p}$ can be identified with the Hankel operator generated by the function

$$
b: \exp (i x) \mapsto-i x+i \pi, \quad x \in[0,2 \pi) .
$$

This function is continuous on $\mathbb{T} \backslash\{1\}$ and has one-sided limits, viz.

$$
b(1-0)=-i \pi, \quad b(1+0)=i \pi,
$$

and its Fourier coefficients $\widehat{b}_{n}, n \in \mathbb{Z}$ are given by

$$
\widehat{b}_{n}= \begin{cases}0, & n=0 \\ 1 / n, & n \neq 0,\end{cases}
$$

where $\mathbb{Z}$ stands for the set of all integers.

Indeed, it is easily seen that the matrix representation of $H(b)$ coincides with $\mathcal{H}_{1}$ and the action of $H(b)$ can be precisely described by $\mathcal{H}_{1}$. For instance,

$$
H(b) t^{n}=\mathcal{H}_{1} t^{n}=\sum_{m=0}^{\infty} \frac{t^{m}}{m+n+1} \in H^{p}(=\operatorname{im} P),
$$

and since

$$
\left.\overline{\left(\mathcal{H}_{1} z^{n}\right.}\right)=\mathcal{H}_{1} t^{n}
$$

the operators $\mathcal{H}_{1}$ is bounded on $H^{p}$ (original definition) and on $\operatorname{im} P=H^{p}$ (equivalent definition). Later on we will sketch the proof of the fact $H(b) \in$ $\mathcal{T}_{p}(P C)$. This result is important because for the algebra $\mathcal{T}_{p}(P C)$ the Fredholm theory is well-developed and the essential spectrum of its elements can be determined (see [7] or [2]). Let us report a few essential features of that 
theory. Notice that the ideal $\mathcal{K}\left(H^{p}\right)$ of all compact operators is contained in $\mathcal{T}_{p}(P C)$, i.e.

$$
\mathcal{K}\left(H^{p}\right) \subset \mathcal{T}_{p}(P C) .
$$

Therefore, we can consider the quotient algebra

$$
\mathcal{T}_{p}^{\pi}(P C):=\mathcal{T}_{p}(P C) / \mathcal{K}\left(H^{p}\right)
$$

Usually, for $A \in \mathcal{T}_{p}(P C)$ the invertibility of the coset $A+\mathcal{K}\left(H^{p}\right)$ in $\mathcal{T}_{p}^{\pi}(P C)$ implies its invertibility in the Calkin algebra $\mathcal{L}\left(H^{p}\right) / \mathcal{K}\left(H^{p}\right)$, and hence the Fredholmness of $A$. The reverse question, does the invertibility of the coset $A+\mathcal{K}\left(H^{p}\right)$ in the Calkin algebra yields its invertibility in $\mathcal{T}_{p}^{\pi}(P C)$ is more interesting. If this is true for every $A \in \mathcal{T}_{p}(P C)$, we say that $\mathcal{T}_{p}^{\pi}(P C)$ is inverse closed in $\mathcal{L}\left(H^{p}\right) / \mathcal{K}\left(H^{p}\right)$.

Let $\overline{\mathbb{R}}$ stand for the two-point compactification of $\mathbb{R}$ and let $\mu_{p}$ and $\nu_{p}$ be the functions defined on $\overline{\mathbb{R}}$ by

$$
\begin{aligned}
& \mu_{p}(\xi):= \begin{cases}\frac{1}{2}\left(1+\operatorname{coth}\left(\pi\left(\xi+\frac{i}{p}\right)\right)\right), & \xi \in \mathbb{R}, \\
0, & \xi=-\infty \\
1, & \xi=\infty,\end{cases} \\
& \nu_{p}(\xi):= \begin{cases}\sinh ^{-1}\left(\pi\left(\xi+\frac{i}{p}\right)\right), & \xi \in \mathbb{R}, \\
0, & \xi= \pm \infty\end{cases}
\end{aligned}
$$

Notice that when $\xi$ runs through the real line from $-\infty$ to $+\infty$, then $\mu_{p}(\xi)$ runs along a circular arc joining the points $z_{1}=0$ to $z_{2}=1$ and passes through the point $(1-\operatorname{coth}(\pi / p)) / 2$.

Theorem 2.2. (cf. Gohberg \& Krupnik [7]).

1. $\mathcal{T}_{p}^{\pi}(P C)$ is a commutative unital Banach algebra.

2. The maximal ideal space of $\mathcal{T}_{p}^{\pi}(P C)$ is homeomorphic to the cylinder $\mathbb{T} \times \overline{\mathbb{R}}$ provided with an exotic non-Euclidian topology.

3. For the algebra $\mathcal{T}_{p}^{\pi}(P C)$, the Gelfand transform $G: \mathcal{T}_{p}^{\pi}(P C) \rightarrow C(\mathbb{T} \times$ $\overline{\mathbb{R}})$ is defined on the generating cosets $T(a)+\mathcal{K}\left(H^{p}\right)$ by

$$
G\left(T(a)+\mathcal{K}\left(H^{p}\right)\right)(t, \xi)=a(t-0)\left(1-\mu_{p}(\xi)\right)+a(t+0) \mu_{p}(\xi) .
$$

4. $\mathcal{T}_{p}^{\pi}(P C)$ is inverse closed in $\mathcal{L}\left(H^{p}\right) / \mathcal{K}\left(H^{p}\right)$.

Corollary 2.3. An operator $A \in \mathcal{T}_{p}(P C)$ is Fredholm if and only if $G(T(a)+$ $\left.\mathcal{K}\left(H^{p}\right)\right)$ does not vanish on $\mathbb{T} \times \overline{\mathbb{R}}$.

Remark 2.4. In the original paper of I. Gohberg and N. Krupnik the parametrization of the mentioned circular arc is different from (2.4).

Let $\mathrm{smb}: \mathcal{T}_{p}(P C) \rightarrow C(\mathbb{T} \times \overline{\mathbb{R}})$ denote the homomorphism

$$
A \mapsto G\left(A+\mathcal{K}\left(H^{p}\right)\right) .
$$

It is worth noting that for $A \in \mathcal{T}_{p}(P C)$ the function smb $A$ is not only responsible for the Fredholmness of $A$ but also allows to determine the index of the 
corresponding operator $A$. For example, let $a: \mathbb{T} \rightarrow \mathbb{C}$ be a piecewise smooth function with finitely many jumps. The range of the function $\operatorname{smb} T(a)$ is a continuous closed curve with a natural orientation. It turns out that for the Fredholm operator $T(a)$, its index can be found by the formula

$$
\text { ind } T(a)=- \text { wind } \operatorname{smb} T(a) \text {, }
$$

where wind $\operatorname{smb} T(a)$ stands for the winding number of the range of $\operatorname{smb} T(a)$, cf. [2, Section 2.73 and Proposition 6.32]. Moreover, following [2, Section 5.49], one can extend the notion of the winding number and the index formula to arbitrary Fredholm operators from $A \in \mathcal{T}_{p}(P C)$, thus obtaining

$$
\text { ind } A=- \text { wind smb } A \text {. }
$$

Definition 2.5. Let $A \in \mathcal{L}\left(H^{p}\right)$. The spectrum of the coset $A+\mathcal{K}\left(H^{p}\right)$ in the Calkin algebra $\mathcal{L}\left(H^{p}\right) / \mathcal{K}\left(H^{p}\right)$, i.e. the set of all complex numbers $\lambda$ such that $\lambda I-A$ is not Fredholm, is called the essential spectrum of $A$ and is denoted by $\operatorname{ess} \operatorname{sp} A$.

Corollary 2.6. For any operator $A \in \mathcal{T}_{p}(P C)$, one has

$$
\text { range smb } A=\operatorname{ess} \operatorname{sp} A \text {. }
$$

\subsection{Toeplitz and Hankel operators on $l^{p}$.}

Let $l^{p}(\mathbb{Z}), 1 \leq p<\infty$ denote the complex Banach space of all sequences $\left(x_{n}\right)_{n \in \mathbb{Z}}$ of complex numbers with the norm

$$
\|x\|_{p}:=\left(\sum_{n \in \mathbb{Z}}\left|x_{n}\right|^{p}\right)^{1 / p}<\infty .
$$

Replacing $\mathbb{Z}$ by the set of all non-negative integers $\mathbb{Z}^{+}$, we get another Banach space, denoted by $l^{p}:=l^{p}\left(\mathbb{Z}^{+}\right)$. The space $l^{p}$ can be seen as a subspace of $l^{p}(\mathbb{Z})$. Let $P$ be the canonical projection of $l^{p}(\mathbb{Z})$ onto $l^{p}$ and $J$ the bounded linear operator on $l^{p}(\mathbb{Z})$ acting by $(J x)_{n}:=x_{-n-1}$. We also consider the operator $Q=I-P$. For a function $a \in L^{\infty}$ the Laurent operator $L(a)$ associated with $a$ acts on the space $l^{0}(\mathbb{Z})$ of all finitely supported sequences on $\mathbb{Z}$ by

$$
(L(a) x)_{k}:=\sum_{m \in \mathbb{Z}} \widehat{a}_{k-m} x_{m}
$$

We note that for every $k$, there are only finitely many non-vanishing summands in this sum. The element $a$ is called a multiplier on $l^{p}(\mathbb{Z})$ if the term

$$
\|L(a)\|:=\sup \left\{\|L(a) x\|_{p}: x \in l^{0}(\mathbb{Z}),\|x\|=1\right\}
$$

is finite. In this case, $L(a)$ extends to a bounded linear operator on $l^{p}(\mathbb{Z})$, which is denoted by $L(a)$ again. The set $M^{p}$ of all multipliers on $l^{p}(\mathbb{Z})$ forms a Banach algebra with the norm

$$
\|a\|_{M^{p}}:=\|L(a)\| .
$$


It is well-known that $M^{2}=L^{\infty}$. Moreover, every function $a$ with bounded total variation $\operatorname{Var}(a)$ is in $M^{p}$ for every $p$, and the Stechkin inequality

$$
\|a\|_{M^{p}} \leq c_{p}\left(\|a\|_{\infty}+\operatorname{Var}(a)\right)
$$

holds with a constant $c_{p}$ independent of $a$. In particular, every trigonometric polynomial and every piecewise constant function on $\mathbb{T}$ are multipliers for every $p$. Let $\mathcal{P}=\mathcal{P}(\mathbb{T})$ and $P K=P K(\mathbb{T})$ be, respectively, the algebra of all trigonometric polynomials and the algebra of all piecewise constant functions on $\mathbb{T}$. By $C_{p}$ and $P C_{p}$ we, respectively, denote the closures of the sets $\mathcal{P}$ and $P K$ in the algebra $M^{p}$. Note that $C_{2}$ is just the algebra of all continuous functions on $\mathbb{T}$, and $P C_{2}$ is the algebra of all piecewise continuous functions on $\mathbb{T}$. Let us finally mention that the Wiener algebra $W$, which consists of all complex-valued functions defined on $\mathbb{T}$ and having absolutely convergent Fourier series, is a subalgebra of $M^{p}$ and

$$
W \subset C_{p} \subset P C_{p} \subset P C .
$$

For these and other properties of multipliers the reader can consult [2].

Let $a \in M^{p}$. The Toeplitz operator $T(a): l^{p} \rightarrow l^{p}$ and the Hankel operator $H(a): l^{p} \rightarrow l^{p}$ thought of as acting on $l^{p}$ are, respectively, defined by

$$
x \mapsto P L(a) x
$$

and

$$
x \mapsto P L(a) J x=P L(a) Q J x .
$$

It is well-known that

$$
\|T(a)\|=\|a\|_{M^{p}}, \quad\|H(a)\| \leq\|a\|_{M^{p}} .
$$

For a subalgebra $A$ of $M^{p}$, let $\mathcal{T}(A)$ refer to the smallest closed subalgebra of $\mathcal{L}\left(l^{p}\right)$, which contains all operators $T(a)$ with $a \in A$. The Fredholm properties of operators from $\mathcal{T}\left(P C_{p}\right)$ are well-understood thanks to the work of R. Duduchava and I. Gohberg/N. Krupnik (see [2] and references therein). Despite Theorems 2.2 and 2.7 are in a sense analogous, their proofs are quite different.

Theorem 2.7. The following assertions are true:

(a) $\mathcal{K}\left(H^{p}\right) \subset \mathcal{T}\left(C_{p}\right) \subset \mathcal{T}\left(P C_{p}\right)$.

(b) $\mathcal{T}^{\pi}\left(P C_{p}\right):=\mathcal{T}\left(P C_{p}\right) / \mathcal{K}\left(l^{p}\right)$ is a commutative unital Banach algebra.

(c) The maximal ideal space of $\mathcal{T}^{\pi}\left(P C_{p}\right)$ is homeomorphic to the cylinder $\mathbb{T} \times \overline{\mathbb{R}}$ provided with an exotic non-Euclidian topology.

(d) For the algebra $\mathcal{T}^{\pi}\left(P C_{p}\right)$ the Gelfand transform $G: \mathcal{T}^{\pi}\left(P C_{p}\right) \rightarrow C(\mathbb{T} \times$ $\overline{\mathbb{R}})$ of the coset $T(a)+\mathcal{K}\left(l^{p}\right)$ is given by

$$
G\left(T(a)+\mathcal{K}\left(l^{p}\right)\right)(t, \xi)=a(t-0)\left(1-\mu_{q}(\xi)\right)+a(t+0) \mu_{q}(\xi),
$$

where $1 / p+1 / q=1$ and $\mu_{q}$ is defined by (2.4) with $p$ replaced by $q$.

(e) The algebra $\mathcal{T}^{\pi}\left(P C_{p}\right)$ is inverse closed in $\mathcal{L}\left(H^{p}\right) / \mathcal{K}\left(H^{p}\right)$. 
We also note that the conclusions mentioned after Theorem 2.2 are also valid for the algebra $\mathcal{T}\left(P C_{p}\right)$. In particular, if $A \in \mathcal{T}\left(P C_{p}\right)$ is Fredholm, then ind $A=-$ wind smb $A$.

\section{The Essential Spectrum of $\mathcal{H}_{\lambda}$ in $H^{p}$ and $l^{p}$}

We start this section with the following simple lemma.

Lemma 3.1. If $\lambda \in \mathbb{C} \backslash \mathbb{Z}_{-}$, then we have:

(a) There is function $b_{\lambda} \in P C_{p}, p \in(1, \infty)$, such that the matrix representation of the Hankel operator $H\left(b_{\lambda}\right)$ acting on $H^{p}$ with respect to standard basis $\left(\chi_{n}\right)_{n \in \mathbb{Z}^{+}}, \chi_{n}(t)=t^{n}, t \in \mathbb{T}$ coincides with the Hilbert matrix $\mathcal{H}_{\lambda}$.

(b) The matrix representation of $H\left(b_{\lambda}\right) \in \mathcal{L}\left(l^{p}\right)$ with respect to the standard basis $\left(e_{n}\right)_{n \in \mathbb{Z}^{+}}$in $l^{p}, e_{n}:=\left(\delta_{n, j}\right)_{j \in \mathbb{Z}^{+}}$and

$$
\delta_{n, j}= \begin{cases}1, & n=j \\ 0, & n \neq j\end{cases}
$$

coincides with $\mathcal{H}_{\lambda}$.

Proof. Considering the residue

$$
\frac{1}{n+\lambda}-\frac{1}{n+1}=\frac{1}{n^{2}}\left(\frac{1-\lambda}{(1+\lambda / n)(1+1 / n)}\right), \quad n \in \lambda \in \mathbb{C} \backslash\left\{-\mathbb{Z}_{+}\right\},
$$

we note that the sequence

$$
\left(\frac{1-\lambda}{(1+\lambda / n)(1+1 / n)}\right)_{n \in \mathbb{N}}
$$

is bounded. Therefore, the function

$$
v_{\lambda}(t):=\sum_{n=1}^{\infty} \frac{1}{n^{2}}\left(\frac{1-\lambda}{(1+\lambda / n)(1+1 / n)}\right) t^{n}, \quad t \in \mathbb{T}
$$

belongs to the Wiener algebra. It is easily seen that the Hankel operator $\mathcal{H}\left(v_{\lambda}\right)$ is compact on both spaces $H^{p}$ and $l^{p}$. Now we set

$$
b_{\lambda}:=b+v_{\lambda},
$$

where $b$ is given by (2.3). Notice that $b_{1}=b \in P C_{p}, 1<p<\infty$. Hence, $H(b)$ is bounded not only on $H^{p}$, but also on $l^{p}$. As a consequence, we get that $\mathcal{H}\left(b_{\lambda}\right)$ is bounded on both spaces $H^{p}$ and $l^{p}, 1<p<\infty$. Clearly, $\mathcal{H}_{\lambda}$ is the matrix representation of $\mathcal{H}\left(b_{\lambda}\right)$ assumed as acting on $H^{p}$ or $l^{p}$, and the lemma is proved.

In what follows we shall identify $\mathcal{H}_{\lambda}$ with $H\left(b_{\lambda}\right)$, the latter acting on $H^{p}$ or $l^{p}$.

The proof of Lemma 3.1 implies the following corollary.

Corollary 3.2. The operator $\mathcal{H}_{\lambda}, \lambda \in \mathbb{C} \backslash \mathbb{Z}_{-}$considered as acting on $H^{p}$ or $l^{p}$ differs from $\mathcal{H}_{1}$ by a compact operator.

Theorem 3.3. Let $1<p<\infty$. The following assertions are true: 
(a) For the operator $\mathcal{H}_{\lambda} \in \mathcal{T}_{p}(P C)$ the Gelfand transform of the coset $\mathcal{H}_{\lambda}+$ $\mathcal{K}\left(H^{p}\right)$ is given by

$$
G\left(\mathcal{H}_{\lambda}+\mathcal{K}\left(H^{p}\right)\right)(t, \xi)=\left\{\begin{array}{ll}
\pi i \nu_{p}(\xi), & t=1 \\
0, & t \neq 1
\end{array},\right.
$$

with the function $\nu_{p}$ defined by (2.5).

(b) For the operator $\mathcal{H}_{\lambda} \in \mathcal{T}\left(P C_{p}\right)$ the Gelfand transform of the coset $\mathcal{H}_{\lambda}+$ $\mathcal{K}\left(l^{p}\right)$ is given by

$$
G\left(\mathcal{H}_{\lambda}+\mathcal{K}\left(l^{p}\right)\right)(t, \xi)=\left\{\begin{array}{ll}
\pi i \nu_{q}(\xi), & t=1 \\
0, & t \neq 1
\end{array} .\right.
$$

(c) The essential spectrum of $\mathcal{H}_{\lambda}$ acting on $H^{p}$ or $l^{p}, 1<p<\infty$ is, respectively, equal to the range of $\pi i \nu_{p}$ or $\pi i \nu_{q}$ where $1 / p+1 / q=1$, and for $p=2$ we have $\operatorname{ess} \operatorname{sp} \mathcal{H}_{\lambda}=[0, \pi]$.

Sketch of the proof. It is well-known that for bounded operators the Fredholm property and index are invariant under compact perturbations. Since $\mathcal{K}\left(H^{p}\right) \subset \mathcal{T}_{p}(P C)$ and $\mathcal{K}\left(l^{p}\right) \subset \mathcal{T}\left(P C_{p}\right)$, the proof of Theorem 3.3 will follow from Theorems 2.2, 2.7 and Corollary 3.2, but some auxiliary results are needed before that.

Identifying $\mathcal{H}_{1}$ with the operator $H(b), b\left(e^{i x}\right)=-i x+i \pi, x \in[0,2 \pi)$, we note that the function $b$ is smooth on $\mathbb{T} \backslash\{1\}$ and has one-sided limits $b(1+0)=i \pi$ and $b(1-0)=-i \pi$. Consider the function

$$
f\left(e^{i x}\right):=\frac{1}{i \pi} b\left(e^{i x}\right)=1-\frac{x}{\pi}, \quad x \in[0,2 \pi),
$$

and $\tilde{f}(t):=f(\bar{t}), t \in \mathbb{T}$. The one-sided limits of these functions at the point $t=1$ are

$$
f(1+0)=\tilde{f}(1-0)=1, \quad f(1-0)=\tilde{f}(1+0)=-1 .
$$

Therefore, $f \widetilde{f}$ is continuous at the point 1 and $f \widetilde{f}(1)=-1$. Since $T(f \widetilde{f})$ and $T(f) T(\widetilde{f})$ are elements of $\mathcal{T}_{p}(P C)$ (and also of $\mathcal{T}\left(P C_{p}\right)$ ), the well-known formula

$$
T(f \widetilde{f})-T(f) T(\tilde{f})=H(f) H(f)
$$

entails

$$
H(f)^{2} \in \mathcal{T}_{p}(P C), \quad\left(H(f)^{2} \in \mathcal{T}\left(P C_{p}\right)\right) .
$$

The Gelfand transform of the coset $H(f)^{2}+\mathcal{K}\left(H^{p}\right)$ equals

$$
G\left(H(f)^{2}+\mathcal{K}\left(H^{p}\right)\right)=G\left(T(f \widetilde{f})-T(f) T(\widetilde{f})+\mathcal{K}\left(H^{p}\right)\right) .
$$

It is easy to compute that

$$
\begin{aligned}
& G\left(T(f \widetilde{f})+\mathcal{K}\left(H^{p}\right)\right)(1, \xi)=-1 \\
& G\left(T(f)+\mathcal{K}\left(H^{p}\right)\right)(1, \xi)=2 \mu_{p}(\xi)-1, \\
& G\left(T(\widetilde{f})+\mathcal{K}\left(H^{p}\right)\right)(1, \xi)=1-2 \mu_{p}(\xi) .
\end{aligned}
$$


Hence

$$
G\left(H(f)^{2}+\mathcal{K}\left(H^{p}\right)\right)(1, \xi)=\left(2 \mu_{p}(\xi)-1\right)^{2}-1=\nu_{p}^{2}(\xi)
$$

and we get

$$
G\left(H(f)^{2}+\mathcal{K}\left(H^{p}\right)\right)(t, \xi)= \begin{cases}\nu_{p}^{2}(\xi), & t=1 \\ 0, & t \neq 1\end{cases}
$$

Notice that these considerations are taken from [22]. What we need to prove now is that the essential spectrum of $H(f)$ coincides with the range of $\nu_{p}$ and $H(f) \in \mathcal{T}_{p}(P C)$. The former follows from the results in $[8,21,22]$. In explicit form they can be found in [20, Section 4.5], which lead to Theorem 4.5.2 in that section and to the description of the essential spectrum of $H(f)$. In order to show that $H(f) \in \mathcal{T}_{p}(P C)$, one can use, for instance, [20, Theorem 2.3.4] and the fact that $\mathcal{T}_{p}(P C)$ can be considered as an subalgebra of $\mathcal{B}(\mathbb{T}, J)$ defined in [20, Section 4.4]. Thus $H(f) \in \mathcal{T}_{p}(P C)$ and

$$
G\left(H(f)+\mathcal{K}\left(H^{p}\right)\right)(t, \xi)= \begin{cases}\nu_{p}(\xi), & t=1, \\ 0, & t \neq 1 .\end{cases}
$$

Since $H(b)=\pi i H(f)$, the assertion (a) of Theorem 3.3 follows.

The assertion (b) of Theorem 3.3 can be proved as follows. Since

$$
G\left(H(f)^{2}+\mathcal{K}\left(l^{p}\right)\right)(t, \xi)= \begin{cases}\nu_{q}^{2}(\xi), & t=1, \\ 0, & t \neq 1,\end{cases}
$$

and $H(f) \in \mathcal{T}\left(P C_{p}\right), 1 / p+1 / q=1$, one can use the results of [17], where the essential spectrum of $H(f)$ in $l^{p}$ is shown to coincide with the range of the function $\nu_{q}$. An alternative proof of these facts can be also found in $[10$, Section 2.4].

The assertion (c) is the consequence of parts (a) and (b) of the present Theorem and Theorems 2.2 and 2.7.

The range of $i \pi \nu_{p}, 1<p<\infty$ is plotted in the next picture and is of drop-like form. Noting that

$$
\nu_{p}(-\xi)=\nu_{q}(\xi), \quad \frac{1}{p}+\frac{1}{q}=1
$$

for all $\xi \in \overline{\mathbb{R}}$, and we have

$$
\operatorname{range}\left(\pi i \nu_{p}\right)=\operatorname{range}\left(\pi i \nu_{q}\right) \text {, }
$$

although the orientation of these curves is different.Therefore, if the range of $\pi i \nu_{p}$ is known, then so is the range $\pi i \nu_{q}, 1 / p+1 / q=1$.

Corollary 3.4. (cf. Dostanic et al. [4]). The norm of $\mathcal{H}_{1}: H^{p} \rightarrow H^{p}$ is equal to $\pi / \sin (\pi / p)$.

Proof. Identifying $\mathcal{H}_{1}$ with the operator $P b J$, we note that $\|b\|_{L^{\infty}}=\pi$ and $\|P\|=1 / \sin (\pi / p)$, cf. [11]. Therefore,

$$
\left\|\mathcal{H}_{1}\right\| \leq\|b\|_{L^{\infty}}\|P\|=\frac{\pi}{\sin (\pi / p)}
$$

On the other hand, the point $\pi / \sin (\pi / p)$ belongs to range $\left(\pi i \nu_{p}\right)=\operatorname{ess} \operatorname{sp} \mathcal{H}_{1}$, and the claim follows. 


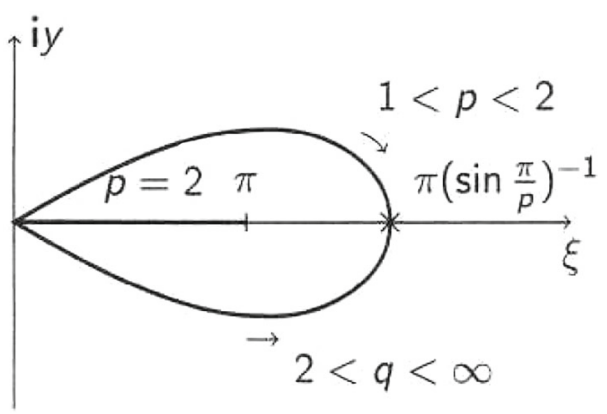

Figure 1. The range of $\pi i \nu_{p}$

Corollary 3.5. The spectral radius of $\mathcal{H}_{1}: H^{p} \rightarrow H^{p}$ is equal to $\pi / \sin (\pi / p)$.

Corollaries 3.4 and 3.5 are also valid for the Hilbert matrix operator $\mathcal{H}_{1}$ considered on the space $l^{p}$.

\section{The Point Spectrum of $\mathcal{H}_{\lambda}$ on $\boldsymbol{H}^{p}$}

Recall that a complex number $\omega$ belongs to the point spectrum of a linear operator $A$ defined on a Banach space $X$ if

$$
\operatorname{ker}_{X}(\omega I-A) \neq\{0\} .
$$

Let $W_{p}$ denote the bounded closed set in $\mathbb{C}$, the boundary of which coincides with the range of the function $\pi i \nu_{p}$ and let $W_{p}^{0}$ be the interior of $W_{p}$. In particular, we have $W_{2}=[0, \pi]$ and $W_{2}^{0}=\varnothing$.

Theorem 4.1. For the operators $\mathcal{H}_{\lambda}$ the following assertions hold:

(a) The operator $\beta I-\mathcal{H}_{\lambda}: H^{p} \rightarrow H^{p}, \lambda \notin \mathbb{Z}_{-}$is Fredholm if and only if $\beta \notin \operatorname{range}\left(\pi i \nu_{p}\right)$. If $\beta \in W_{p}^{0}$ and $\beta I-\mathcal{H}_{\lambda}$ is Fredholm, then

$$
\begin{array}{llrl}
\operatorname{ind}_{H^{p}}\left(\beta I-\mathcal{H}_{\lambda}\right) & =1, & & 1<p<2, \\
\operatorname{ind}_{H^{p}}\left(\beta I-\mathcal{H}_{\lambda}\right) & =-1, & & 2<p<\infty .
\end{array}
$$

(b) The operator $\beta I-\mathcal{H}_{\lambda}: l^{p} \rightarrow l^{p}, \lambda \neq \mathbb{Z}_{-}$is Fredholm if and only if $\beta \notin \operatorname{range}\left(\pi i \nu_{q}\right)$. If $\beta \in W_{p}^{0}$ and $\beta I-\mathcal{H}_{\lambda}$ is Fredholm, then

$$
\begin{aligned}
\operatorname{ind}\left(\beta I-\mathcal{H}_{\lambda}\right) & =-1, & & 1<p<2, \\
\operatorname{ind}\left(\beta I-\mathcal{H}_{\lambda}\right) & =1, & & 2<p<\infty .
\end{aligned}
$$

(c) If $\beta \notin W_{p}, 1<p<\infty$, then the operator $\beta I-\mathcal{H}_{\lambda}$ acting on $H^{p}$ or $l^{p}$ is Fredholm with index zero. If $\lambda>1 / 2$ is real, then $\beta I-\mathcal{H}_{\lambda}$ is invertible on both spaces $H^{p}$ and $l^{p}$.

Proof. Since $\mathcal{H}_{\lambda}$ differs from $\mathcal{H}_{1}$ by a compact operator and compact perturbations do not influence Fredholmness and index, it suffices to prove the assertions (a) and (b) for $\lambda=1$. 
(a) As was mentioned in Sect. 2, the index of the Fredholm operator $\beta I-\mathcal{H}_{1}$ can be expressed by minus the winding number of the oriented arc $\beta-\pi i \nu_{p}(\xi)$. For $1<p<2(2<p<\infty)$ and $\beta \in W_{p}^{0}$ this oriented arc has winding number $1(-1)$. Thus (a) is proven.

(b) The proof of assertion (b) is analogous, but one has to use the function $\nu_{p}(-\xi)=\nu_{q}(\xi), 1 / p+1 / q=1$ instead of $\nu_{p}(\xi)$.

(c) Theorem 1.1 shows that the spectrum of the selfadjoint operator $\mathcal{H}_{\lambda}$, $\lambda \geq 1 / 2$ on $l^{2}$ is $[0, \pi]$. Therefore, if $\beta \notin[0, \pi]$, the operator $\beta I-$ $\mathcal{H}_{\lambda}: H^{2} \rightarrow H^{2}$ is invertible. It is easily seen that if $\beta \notin W_{p}, p \neq 2$, then $\beta I-\mathcal{H}_{\lambda}$ is Fredholm with index zero and $\beta \notin[0, \pi]$. Hence, both operators $\beta I-\mathcal{H}_{\lambda}$ on $H^{2}$ and $\beta I-\mathcal{H}_{\lambda}$ on $H^{p}$ are Fredholm with index zero. Since $H^{2}$ is dense in $H^{p}$ for $p<2$ and $H^{p}$ is dense in $H^{2}$ for $p>2$, the kernels of these operators coincide by Lemma 2.1 , hence are trivial. The same argumentation also works for $l^{p}$-spaces.

Corollary 4.2. Let $\lambda \notin \mathbb{Z}_{-}$.

(a) If $1<p<2$, then $W_{p}^{0}$ belongs to the point spectrum of the operator $\mathcal{H}_{\lambda}: H^{p} \rightarrow H^{p}$.

(b) If $2<p<\infty$, then $W_{p}^{0}, p \neq 2$ belongs to the point spectrum of the operator $\mathcal{H}_{\lambda}: l^{p} \rightarrow l^{p}$.

Proof. The proof follows from Theorem 4.1.

Corollary 4.3. Let $\mu \in \mathbb{C} \backslash\{0\}$ and $\mathcal{H}_{\lambda}-\mu I$ acts on $H^{p}$ or $l^{p}$. Then

$$
\operatorname{dim} \operatorname{ker}\left(\mathcal{H}_{\lambda}-\mu I\right)<\infty \text {. }
$$

Proof. We give the proof for operators acting on $H^{p}$. If $\mu \notin \operatorname{range}\left(\pi i \nu_{p}\right)$, then $\mathcal{H}_{\lambda}-\mu I$ is Fredholm by Theorem 4.1 (a). If $\mu \in \operatorname{range}\left(\pi i \nu_{p}\right)$, we choose a number $s, 1<s<2$ such that

$$
\begin{array}{lll}
-s^{-1}<\min \{-1 / p,-1 / 2\} & \text { for } \quad p \leq 2 \\
-s^{-1}<\min \{-1 / q,-1 / 2\} & \text { for } \quad 2<p, \quad 1 / p+1 / q=1 .
\end{array}
$$

In both cases, $\mu \in W_{s}^{0}$ and $\mathcal{H}_{\lambda}-\mu I$ is Fredholm on $H^{s}$. Since $H^{s} \supset H^{p}$, it yields the claim for $\mu \in \operatorname{range}\left(\pi i \nu_{p}\right)$.

Remark 4.4. Corollary 4.3 is also in force for $\mu=0$. We shall show later on that $\operatorname{dim} \operatorname{ker} \mathcal{H}_{\lambda}=0$, cf. Lemma 4.18 .

Let us now draw attention to other points of interest, viz.

(I) Are there any other points in the point spectrum?

(II) What can be said about eigenvectors and their multiplicities?

The answers to these questions are particularly hidden in Hill's paper [9]. In addition to Theorem 1.3 he proved the following result.

Theorem 4.5. (I) Every complex number $M$ with positive real part is a latent root of $\mathcal{H}_{\lambda}$ and

$$
M=\frac{\pi}{\sin (\pi \mu)}, \quad 0<R e(\mu) \leq \frac{1}{2} .
$$


(II) Consider the complex number

$$
\Lambda:=\frac{\pi}{\sin (\lambda \pi)} .
$$

Any latent root $\kappa \neq \pm \Lambda$ of $\mathcal{H}_{\lambda}$ has multiplicity 1 , that is the related root vectors form a one-dimensional space.

(III) If $\lambda>0$, then all latent root of $\mathcal{H}_{\lambda}$ have positive real parts and all latent roots, including $|\Lambda|$, have multiplicity 1 . If $\lambda<0, \lambda \notin \mathbb{Z}_{-}$, then all latent roots except $-|\Lambda|$, have positive real parts.

Remark 4.6. 1. The eigenspace associated with the latent root $M$, which has a positive real part, contains the vector $\left(x_{0}(\lambda, \mu), x_{1}(\lambda, \mu), \ldots\right)$ by Theorem 1.4, where $\mu \in(0,1 / 2]$ is defined by the equation $M=\pi /$ $(\sin (\pi \mu))$.

2. If $M$ is a latent root with positive real part and $M \notin\{\Lambda,-\Lambda\}$, then by Theorem 4.5 the related eigenspace is spanned by the vector

$$
\left(x_{0}(\lambda, \mu), x_{1}(\lambda, \mu), \ldots\right),
$$

where $\mu$ is defined by $M=\pi /(\sin (\pi \mu)$, and $\operatorname{Re}(\mu) \in(0,1 / 2]$. Suppose that $1 / q<\operatorname{Re}(\mu) \leq 1 / 2$ and therefore $-1 / p<-1+\mu \leq-1 / 2$. Then by Theorem $4.1(\mathrm{a})$, the number $M$ belongs to $M_{p}^{0}$, that is $M$ is an eigenvalue of $\mathcal{H}_{\lambda}: H^{p} \rightarrow H^{p}, 1<p<2$. Moreover, there exists an eigenfunction $h$ such that $\left(M I-\mathcal{H}_{\lambda}\right) h=0, h \neq 0$. Using the matrix representation of $M I-\mathcal{H}_{\lambda}$ with respect to Schauder basis $\left\{\chi_{n}\right\}_{n \in \mathbb{Z}_{+}}$, we see that the vector $\left(\widehat{h}_{0}, \widehat{h}_{1}, \ldots\right)$ formed by the Fourier coefficients $\widehat{h}_{n}$ of $h, n \in \mathbb{Z}_{+}$is a root vector for the latent root $M$. Hence, there is a complex number $\eta \neq 0$ such that

$$
\left(x_{0}(\lambda, \mu), x_{1}(\lambda, \mu), \ldots\right)=\eta\left(\widehat{h}_{0}, \widehat{h}_{1}, \ldots\right) .
$$

Consequently,

$$
\sum_{n=0}^{\infty} x_{n}(\lambda, \mu) z^{n}=\eta h(z)
$$

and the radius of convergence of the above series is at least one. Here we used the fact the Taylor and Fourier coefficients of $h$ coincide. Notice also that if $M \in M_{p}^{0}$ is fixed, then the associated $\mu$ does not depend on $\lambda$.

3. It is not claimed that $\pm|\Lambda|$ are latent roots, since for $\lambda=0,-1, \ldots$ the Hilbert matrix is not defined and for $\lambda \in \mathbb{N}$, the function $\pi / \sin (\pi \lambda)$ is not defined. However, if any of $\pm|\Lambda|$ is a latent root, then the linear multiplicity of the corresponding root can exceed one, cf. Theorem 1.3.

4. Claim (I) can be easily obtained from Corollary 4.2(a).

For further analysis we introduce the strip

$$
\Omega:=\left\{a \in \mathbb{C}:-1<\operatorname{Re}(a) \leq-\frac{1}{2}\right\} .
$$


As a first motivation for introduction of this set, we refer Theorem 1.3. Thus the parameter $\mu$ in Hill's paper [9], cf. Theorem 1.3, has to satisfy the inequality $0<\mu \leq 1 / 2$. Therefore, $a:=\mu-1 \in \Omega$, and

$$
\frac{\pi}{\sin (\pi \mu)}=-\frac{\pi}{\sin (\pi a)}=\frac{\pi i}{\sinh (\pi(\xi+i / s))},
$$

where $a=-1 / s+i \xi$ with $1<s \leq 2$ and $\xi \in \mathbb{R}$. Moreover, the function

$$
z \mapsto \frac{\pi i}{\sinh (-\pi i z)}
$$

maps the line $-1 / s+i \xi$ onto the arc range $\left.\left(\pi i \nu_{s}\right) \backslash\{0\}\right)$ and

$$
\lim _{\xi \rightarrow \pm \infty} \frac{\pi i}{\sinh (\pi(\xi+i / s))}=0
$$

Recall that $\pi / \sin (\pi \mu)$ is a latent root of $\mathcal{H}_{\lambda}$ by Theorem 1.3.

As was already mentioned, the natural orientation of $\overline{\mathbb{R}}$ induces an orientation of $\pi i \nu_{s}$. The observations above suffice to describe the spectrum of $\mathcal{H}_{1}$ on $H^{p}$ and $l^{p}$. However, we postpone it because we want to consider a more general situation.

A more complete study of the point spectrum of $\mathcal{H}_{\lambda}$ on $H^{p}$ for $\lambda \in \mathbb{C} \backslash \mathbb{Z}$ was presented by Aleman et al. [1]. The restriction $\lambda \in \mathbb{C} \backslash \mathbb{Z}$ is caused by the fact that in [1], the matrix $\mathcal{H}_{\lambda}$ is represented as an integral operator acting on the space $H^{p}$, viz.

$$
\left(\mathcal{H}_{\lambda} f\right)(z):=\frac{1}{\kappa} \int_{\gamma} \frac{f(t) t^{\lambda-1}}{1-t z} d t,
$$

where $\kappa=e^{2 \pi i \lambda}-1$ and $\gamma$ is the positively oriented boundary of the Stolz angle

$$
\{z \in \mathbb{D}:|1-z| \leq \sigma(1-|z|)\}, \quad \sigma>1 .
$$

It is clear that the representation (4.1) is valid only for $\lambda \in \mathbb{C} \backslash \mathbb{Z}$, since $e^{2 \pi i n}-1=0$ for $n \in \mathbb{Z}_{+}$. The key observation in this paper is that $\mathcal{H}_{\lambda}$ "almost commutes" with two specific second order linear differential operators having polynomial coefficients, namely with the operators

$$
\left(D_{1, \lambda} f\right)(z):=\left(z^{2}-1\right) f^{\prime}(z)+\lambda z f(z)
$$

and

$$
\left(D_{2, \lambda} f\right)(z):=z(z-1)^{2} f^{\prime \prime}(z)+(z-1)[(\lambda+2) z-\lambda z] f^{\prime}(z)+\lambda z f(z),
$$

defined on the set of all functions $f$ analytic on $\mathbb{D}$. More precisely, the following theorem is true.

Theorem 4.7. (cf. Aleman et al. [1, Theorem 3.1]). For any $f \in H^{p}$ and $\lambda \in \mathbb{C} \backslash \mathbb{Z}$, the operators $D_{1, \lambda}$ and $D_{2, \lambda}$ satisfy the equations

$$
\begin{aligned}
& \left(\mathcal{H}_{\lambda} D_{1, \lambda}-D_{1, \lambda} \mathcal{H}_{\lambda}\right) f=\frac{\lambda-1}{\kappa} \int_{\gamma} f(t) t^{\lambda-2} d t, \\
& \left(\mathcal{H}_{\lambda} D_{2, \lambda}-D_{2, \lambda} \mathcal{H}_{\lambda}\right) f=0 .
\end{aligned}
$$


We also note that the functional $\Phi_{\lambda}$ defined by

$$
f \mapsto \frac{1}{\kappa} \int_{\gamma} f(t) t^{\lambda-2} d t,
$$

plays an important role. It is clear that $\Phi_{\lambda}(f)$ is equal to $\left(\mathcal{H}_{\lambda-1} f\right)(0)$. The Eqs. (4.2) and (4.3) are corner stones in the study of the point spectrum of the operator $\mathcal{H}_{\lambda}, \lambda \in \mathbb{C} \backslash \mathbb{Z}$ on $H^{p}$. One reason for that is because the eigenvalue problem

$$
\begin{aligned}
& D_{1, \lambda} f=\nu f, \\
& D_{2, \lambda} f=\nu f,
\end{aligned}
$$

for functions $f$ analytic on $\mathbb{D}$, can be studied. The eigenvalue problem (4.4) has analytic solutions [1, Proposition 3.2], which are constant multipliers of

$$
f_{\lambda}(z)=(1-z)^{(-\lambda+\nu) / 2}(1+z)^{(-\lambda-\nu) / 2}
$$

Notice that we can always define a branch of the first factor on the right-hand side on $\mathbb{C} \backslash[1, \infty)$ and a branch of the second factor on $\mathbb{C} \backslash(-\infty, 1]$.

The eigenvalue problem (4.5) is more involved. In order to formulate the related results, we have to consider the hypergeometric series

$$
F(\alpha, \beta ; \gamma ; z):=\sum_{k=0}^{\infty} \frac{(\alpha)_{k}(\beta)_{k}}{(\gamma)_{k}} z^{k},
$$

where the parameters $\alpha, \beta$ and $\gamma$ can take any real or complex values provided that $\gamma \neq \mathbb{Z}_{-}$and the symbol $(\tau)_{k}, k \in \mathbb{Z}$ denotes the numbers $(\tau)_{0}=1$ and $(\tau)_{k}=\tau(\tau+1) \ldots(\tau+k-1), k \neq 0$. The series (4.6) becomes a polynomial if either $\alpha$ or $\beta$ belongs to $\mathbb{Z}_{-}$. For all other choices of $\alpha$ and $\beta$, the radius of convergence of (4.6) equals one.

In what follows, the solutions $a$ and $a^{\prime}$ of the quadratic equation

$$
x^{2}+x+\lambda-\nu=0
$$

play an important role. We point out that for any $a \in \mathbb{C}$ there is a $\nu \in \mathbb{C}$ such that $a$ is a solution of Eq. (4.7).

Theorem 4.8. (cf. Aleman et al. [1]). For $\lambda \in \mathbb{C} \backslash \mathbb{Z}$ and $\nu \in \mathbb{C}$, the solutions of the eigenvalue problem

$$
D_{2, \lambda} f=\nu f, \quad f \text { is analytic in } \mathbb{D},
$$

form a one-dimensional space spanned by

$$
\begin{aligned}
f_{a}(z) & =(1-z)^{a} F(a+1, a+\lambda ; \lambda ; z) \\
& =(1-z)^{a^{\prime}} F\left(a^{\prime}+1, a^{\prime}+\lambda ; \lambda ; z\right)=f_{a}^{\prime}(z),
\end{aligned}
$$

where $a$ and $a^{\prime}$ are the solutions of equation (4.7). Moreover, if $a$ and $a^{\prime}$ are ordered as $\operatorname{Re}\left(a^{\prime}\right) \geq-1 / 2 \geq \operatorname{Re}(a)$, one has:

(i) If $\operatorname{Re}(a)<-1 / 2$ and $a+1-\lambda=-a^{\prime}-\lambda \notin \mathbb{Z}_{+}$, then the limit

$$
\lim _{x \rightarrow 1-0}(1-x)^{-\operatorname{Re}(a)}\left|f_{a}(x)\right|
$$

exists, is finite and not equal to zero ( $x$ real). Moreover, if $f_{a} \in H^{p}$, then $-1 / p<\operatorname{Re}(a)$. 
(ii) If $\operatorname{Re}(a)<-1 / 2$ and $\lambda-a-1=a^{\prime}+\lambda=-n, n \in \mathbb{Z}_{+}$, then $\operatorname{Re}(\lambda)<1 / 2$ and

$$
f_{a^{\prime}}(z)=(1-z)^{a^{\prime}} Q(z),
$$

where $Q(z):=F\left(a^{\prime}+1, a^{\prime}+\lambda ; \lambda ; z\right)$ is a polynomial of degree $n$.

(iii) If Re $a=-1 / 2$ and if $\lambda-a^{\prime}-1 \notin \mathbb{Z}_{-}$, then

$$
\left|f_{a}(x)\right| \leq M(1-x)^{-1 / 2}\left(1+\log \left(\frac{1}{1-x}\right)\right), \quad x \in[0,1),
$$

where $M$ is a constant, which does not depend on $x$. Moreover, $f_{a} \in H^{p}$, $1<p<2$, but $f_{a} \notin H^{2}$.

(iv) If Re $a=-1 / 2$ and $\lambda-a^{\prime}-1 \in \mathbb{Z}_{-}$, then

$$
f_{a}(z)=(1-z)^{-\lambda-n} F(-\lambda-n+1,-n ; \lambda ; z)
$$

belongs to $H^{p}, 1<p<2$ and $-1 / p<R e(-\lambda)-n$, but not to $H^{2}$.

A few comments are in order. The fact that the solution of equation $D_{2, \lambda} f=\nu f$ is independent of the choice of the root of the equation (4.7) is known as Euler's formula (see [14, p. 248, formula(9.5.3)]).

Assertion (i) is a consequence of the well-known Gauss' formula

$$
\lim _{x \rightarrow 1^{-}} F(a+1, a+\lambda ; \lambda ; x)=\frac{\Gamma(\lambda) \Gamma(-2 a-1)}{\Gamma(\lambda-a-1) \Gamma(-a)} \neq 0,
$$

if $\lambda-a-1 \notin \mathbb{Z}_{-}$and $\operatorname{Re}(-2 a-1)>0$ (see also [14, Section 9.3]). The last claim in (i) is a consequence of the Fejer-Riesz inequality.

Assertion (ii) follows directly from (4.6). To prove (iii) we need to study the behaviour of the coefficients $\xi_{n}$ of $F(a+1, a+\lambda ; \lambda ; z)$.

Using

$$
(\eta)_{k}=\frac{\Gamma(\eta+k)}{\Gamma(\eta)}
$$

one gets for the $n$-th coefficient $\xi_{n}, n \geq 1$ that

$$
\begin{aligned}
\left|\xi_{n}\right| & =\left|\frac{(1 / 2+i \operatorname{Im} a)_{n}(-1 / 2+i \operatorname{Im} a+\lambda)_{n}}{n !(\lambda)_{n}}\right| \\
& =\left|\frac{\Gamma(1 / 2+i \operatorname{Im} a+n)}{\Gamma(1+n)} \cdot \frac{\Gamma(-1 / 2+i \operatorname{Im} a+\lambda+n)}{\Gamma(\lambda+n)} \cdot W\right| \\
& =n^{-1}\left(1+\frac{C}{n}+\mathcal{O}\left(\frac{1}{n^{2}}\right)\right)|W| \leq M n^{-1},
\end{aligned}
$$

where $M$ and $C$ do not depend on $n$ and

$$
W=\frac{\Gamma(\lambda)}{\Gamma(1 / 2+i \operatorname{Im} a) \Gamma(-1 / 2+i \operatorname{Im} a+\lambda)} .
$$

The estimate (4.11) is a consequence of the representation

$$
\frac{\Gamma\left(n+z_{1}\right)}{\Gamma\left(n+z_{2}\right)}=n^{z_{1}-z_{2}}\left[1+\frac{\left(z_{1}-z_{2}\right)\left(z_{1}+z_{2}-1\right)}{2 n}+\mathcal{O}\left(\frac{1}{n^{2}}\right)\right],
$$

where $z_{1}$ and $z_{2}$ are arbitrary complex constants such that $\Gamma\left(n+z_{1}\right)$ and $\Gamma\left(n+z_{2}\right)$ are defined (see $[14$, p. 15 , Problem 7$\left.]\right)$. The conclusion $\lambda-a-1=$ 
$\lambda+a \notin \mathbb{Z}_{-}$insures that $\Gamma(a+\lambda+1+n)$ and $\Gamma(a+\lambda+n)$ are defined. If we expand $1+\log (1 /(1-x))$ in a power series, we get

$$
1+\log \left(\frac{1}{1-x}\right)=1+\sum_{n=1}^{\infty} \frac{1}{n} x^{n}
$$

and arrive finally at

$$
|F(a+1, a+\lambda ; \lambda ; x)| \leq M\left|1+\log \left(\frac{1}{1-x}\right)\right|, \quad x \in[0,1),
$$

so that (4.9) follows. The last claim in (iii) is a consequence of the Fejer-Riesz inequality. The proof of (iv) can be carried out similar to the proof of (d), Theorem 4.11.

Theorem 4.8 is one of the corner stones in description of the point spectrum of the operator $\mathcal{H}_{\lambda}: H^{p} \rightarrow H^{p}$. Another key result is the following lemma.

Lemma 4.9. (cf. Aleman et al. [1, Lemma 4.4]). Let $\tau \in \mathbb{C} \backslash\{0\}, f \in \operatorname{ker}\left(\mathcal{H}_{\lambda}-\right.$ $\tau I), f \neq 0$, and $\mathcal{H}_{\lambda}: H^{p} \rightarrow H^{p}, p \geq 2$ or $f \in H^{\infty}$. Then $\operatorname{im}\left(\mathcal{H}_{\lambda}-\tau I\right)$ does not contain constant functions. In particular,

$$
\int_{\gamma} f(t) t^{\lambda-2} d t=0
$$

and $D_{1, \lambda} f \in \operatorname{ker}\left(\mathcal{H}_{\lambda}-\tau I\right)$.

We also need several times the following lemma.

Lemma 4.10. (cf. Aleman et al. [1, Theorem 2.1(i)]). Let $f$ be analytic in $\mathbb{D}$, integrable on $[0,1]$ and let $\mathcal{H}_{\lambda}$ be as in (4.1). If $f \in L^{p}[0,1] 1<p<\infty$, then $\mathcal{H}_{\lambda} f \in H^{p}$.

The above preparations are needed for the comments made after the following theorem, which in fact is the main result of [1].

Theorem 4.11. (cf. Aleman et al. [1]). Let $\lambda \in \mathbb{C} \backslash \mathbb{Z}$.

(a) Let $p \geq 2$ and $(1-z)^{-\lambda} \in H^{p}$, i.e. Re $(\lambda)<1 / p$. If $N \geq 0$ is the largest integer such that $(1-z)^{-N-\lambda} \in H^{p}$, then

$$
f_{n}(z):=(1-z)^{-n-\lambda}(1+z)^{n}, \quad 0 \leq n \leq N,
$$

are eigenfunctions of $\mathcal{H}_{\lambda}$ related to the eigenvalues $(-1)^{n} \pi /(\sin (\lambda \pi))$.

(b) For any $p \geq 2$ set

$$
\begin{aligned}
& \mathcal{V}_{e}:=\left\{f_{n} \in H^{p}: 0 \leq n \leq N, n \text { is even }\right\}, \\
& \mathcal{V}_{o}:=\left\{f_{n} \in H^{p}: 0<n \leq N, n \text { is odd }\right\} .
\end{aligned}
$$

Then the systems $\mathcal{V}_{e}$ and $\mathcal{V}_{o}$ are bases of the kernels of $\mathcal{H}_{\lambda}-(\pi / \sin (\pi \lambda)) I$ and $\mathcal{H}_{\lambda}+(\pi / \sin (\pi \lambda)) I$, respectively. Moreover, every eigenfunction of $\mathcal{H}_{\lambda}$ belongs to $\operatorname{ker}\left(\mathcal{H}_{\lambda}-(\pi / \sin (\pi \lambda)) I\right)$ or to $\operatorname{ker}\left(\mathcal{H}_{\lambda}+(\pi / \sin (\pi \lambda)) I\right)$. 
(c) If $p<2$, then the point spectrum of $\mathcal{H}_{\lambda}$ is the image of the set

$$
\left\{-\frac{1}{p}<\operatorname{Re}(a) \leq \frac{1}{2}\right\} \cup \Omega_{\lambda}
$$

under the map $a \mapsto-\pi /(\sin (\pi a))$ or, equivalently, under the map $a \mapsto$ $(\pi i) /(\sinh (-\pi i a))$. The set $\Omega_{\lambda}$ is by definition

$\left\{\xi \in\{-\lambda,-\lambda-1\}:-\frac{\pi}{\sin (\pi \xi)}\right.$ is in the point spectrum of $\left.\mathcal{H}_{\lambda}: l^{2} \rightarrow l^{2}\right\}$.

Each eigenvalue $-\pi /(\sin (\pi a)), a \neq-\lambda,-\lambda-1$ has multiplicity one and the corresponding eigenspace is spanned by

$$
f_{a}(z)=(1-z)^{a} F(a+1, a+\lambda ; \lambda ; z) .
$$

(d) Let $n \in \mathbb{Z}_{+}$be such that $-1 / 2 \leq \operatorname{Re}(\lambda)+n-1<0$. If $-1 / p<\operatorname{Re}(-\lambda)-$ $n$, then $\mathcal{H}_{\lambda}: H^{p} \rightarrow H^{p}$ owns an eigenfunction, viz.

$$
g(z):=(1-z)^{-\lambda-n} F(-\lambda-n+1,-n ; \lambda ; z)
$$

with the eigenvalue

$$
(-1)^{n} \frac{\pi}{\sin (\pi \lambda)}
$$

Moreover, $g \notin H^{2}$.

The proof of this theorem given in [1] is relatively complex. So we restrict ourselves to comments concerning the claim (c) and provide independent proofs for the claims (a), (b) and (d).

Starting with the case $p<2$, i.e. with the assertion (c), we note that $\Omega_{\lambda}$ contains at most two elements. Now let $-1 / p<\operatorname{Re}(a)<-1 / 2$ and $a+1-\lambda=-a^{\prime}-\lambda \notin \mathbb{Z}_{+}$. By Theorem 4.8(i), the function $f_{a}$ belongs to $H^{p}$ only if $-1 / p<\operatorname{Re} a$. Since the restriction of $f_{a}$ to $[0,1]$ belongs to $L^{p}[0,1]$, then $\mathcal{H}_{\lambda} f_{a}$ belongs to $H^{p}$ by Lemma 4.10 . Let $\xi \in \mathbb{C}$ be such that $a, \operatorname{Re}(a)<-1 / 2$ is a root of the Eq. (4.7). Taking into account the Eq. (4.3), we write

$$
\left(D_{2, \lambda}-\xi I\right) \mathcal{H}_{\lambda} f_{a}=\mathcal{H}_{\lambda}\left(D_{2, \lambda}-\xi I\right) f_{a}=0,
$$

so that $\mathcal{H}_{\lambda} f_{a}$ belongs to the space $\operatorname{ker}\left(D_{2, \lambda}-\xi I\right)$, which has dimension one by Theorem 4.8. Thus

$$
\mathcal{H}_{\lambda} f_{a}=\mu f_{a}
$$

for a $\mu \in \mathbb{C}$, and the equation $f_{a}(0)=1$ yields

$$
\mu=\left(\mathcal{H}_{\lambda} f_{a}\right)(0)=\frac{1}{\kappa} \int_{\gamma} t^{\lambda-1} f_{a}(t) d t
$$

It is shown in [1] that if $\operatorname{Re}(y)>0$ and $\Gamma(x), \Gamma(y)$ and $\Gamma(x+y)$ are defined, then

$$
\frac{1}{\kappa} \int_{\gamma} t^{\lambda-1}(1-t)^{y-1} d t=\frac{\Gamma(x) \Gamma(y)}{\Gamma(x+y)},
$$

and

$$
\left(\mathcal{H}_{\lambda} f_{a}(0)=\Gamma(a+1) \Gamma(-a)=-\frac{\pi}{\sin (\pi a)}\right.
$$


Hence, the equation

$$
\mathcal{H}_{\lambda} f_{a}=-\frac{\pi}{\sin (\pi a)} f_{a}
$$

holds under the condition $a+1-\lambda \notin \mathbb{Z}_{+}$.

Assume now that the $\operatorname{Re}(a)=-1 / 2$. Then the estimate (4.9) and $[1$, Theorem 2.1(i)] imply that

$$
\mathcal{H}_{\lambda} f_{a}=-\frac{\pi}{\sin (\pi a)} f_{a} \in H^{p}, \quad 1<p<2
$$

An independent proof of this result can be given using Corollary 4.2(a).

Finally, we assume that $\operatorname{Re}(a)<-1 / 2$ and $\lambda-a-1=a^{\prime}+\lambda=-n$, $n \in \mathbb{Z}_{+}$. Notice that we do not suppose that $\operatorname{Re}(a)>-1$. Since $H^{p} \supset H^{2}$ for $p<2$, the set $\Omega_{\lambda}$ is included in the point spectrum of $\mathcal{H}_{\lambda}$. However, it is worth noting that if $\operatorname{Re}(\lambda)>1 / 2$, then $\Omega_{\lambda}$ can be an empty set and if $\operatorname{Re}(\lambda) \leq 1 / 2$, it can contain one or two elements.

Now we consider case (a). If the condition $\operatorname{Re}(a)<-1 / 2, \lambda-a-1=$ $a^{\prime}+\lambda=-n, n \in \mathbb{Z}_{+}$is fulfilled and $N \geq 0$ is the largest integer such that $(1-z)^{-\lambda-N} \in H^{p}, 2 \leq p<\infty$, then $f_{a}=f_{a^{\prime}}, a^{\prime}=-\lambda-n$ and

$$
f_{a^{\prime}}(z)=(1-z)^{-\lambda-n} F(-\lambda-n+1,-n ; \lambda ; z)=(1-z)^{-\lambda-n} Q_{n}(z),
$$

where $Q_{n}(z)$ is a polynomial of degree $n$. More generally, if $\lambda$ is fixed, then for $0 \leq k \leq N$ the function

$$
g_{k}(z)=(1-z)^{-\lambda-k} F(-\lambda-k+1,-k ; \lambda ; z)
$$

belongs to $H^{p}$ and

$$
\mathcal{H}_{\lambda} g_{k}=-\frac{\pi}{\sin (\pi(-\lambda-k))} g_{k}=(-1)^{k} \frac{\pi}{\sin (\pi \lambda)} g_{k}
$$

Thus for $\mathcal{H}_{\lambda}: H^{p} \rightarrow H^{p}, p \geq 2,0 \leq k \leq N$, and $\Lambda:=\pi / \sin (\pi \lambda)$, we have that

$$
g_{k} \in M_{\Lambda}:=\operatorname{ker}\left(\mathcal{H}_{\lambda}-\Lambda I\right) \quad \text { if } \quad k \quad \text { is even }
$$

and

$$
g_{k} \in M_{-\Lambda}:=\operatorname{ker}\left(\mathcal{H}_{\lambda}+\Lambda I\right) \quad \text { if } \quad k \quad \text { is odd }
$$

Clearly, $N$ depends on $p$, and Lemma 4.9 yields $\Phi_{\lambda}\left(g_{k}\right)=0$ for all $k \in[0, N]$. It can be proven that the systems

$$
\begin{aligned}
& \mathcal{U}_{e}:=\left\{g_{k}: 0 \leq k \leq N \text { and } k \text { is even }\right\}, \\
& \mathcal{U}_{o}:=\left\{g_{k}: 0<k \leq N \text { and } k \text { is odd }\right\},
\end{aligned}
$$

are linearly independent (see Remark 4.16). We note that the systems $\mathcal{U}_{e}, \mathcal{U}_{o}$ differ from the system (4.12) used in [1]. Lemma 4.14 below may serve as a hint, why that system is of relevance. We like to present a transparent and relatively simple proof of the assertion. It is based on the following lemma used in [1], but for different aims. Thus we introduce the forward shift $V: H^{p} \rightarrow H^{p}$ by $h \mapsto z h$, i.e. $V$ is the multiplication operator by $z$. 
Lemma 4.12. Let $\lambda \in \mathbb{C} \backslash \mathbb{Z}$ and $\Phi_{\lambda}: H^{p} \rightarrow \mathbb{C}$ be the liner functional defined by

$$
h \mapsto \frac{1}{\kappa} \int_{\gamma} h(t) t^{\lambda-2} d t .
$$

Then we have

$$
\begin{aligned}
& V \mathcal{H}_{\lambda}=-\Phi_{\lambda}+\mathcal{H}_{\lambda-1}, \\
& \mathcal{H}_{\lambda}=H_{\lambda-1} V .
\end{aligned}
$$

Proof. Let us show the formula (4.14), for example. One can write

$$
\begin{aligned}
& \frac{z f(t) t^{\lambda-1}}{1-t z}=\frac{(-1+t z+1) f(t) t^{\lambda-2}}{1-t z} \\
= & -f(t) t^{\lambda-2}+\frac{f(t) t^{\lambda-2}}{1-t z},
\end{aligned}
$$

and the Eq. (4.14) follows.

The Eqs. (4.14) and (4.15) imply

$$
\begin{aligned}
& H_{\lambda-1}(V-I)+\Phi_{\lambda}=(I-V) \mathcal{H}_{\lambda}, \\
& H_{\lambda-1}(V+I)-\Phi_{\lambda}=(I+V) \mathcal{H}_{\lambda} .
\end{aligned}
$$

Corollary 4.13. Suppose that $f \in H^{p}$ is an eigenfunction of $\mathcal{H}_{\lambda}$ with eigenvalue $\tau$ and $\Phi_{\lambda}(f)=0$. Then

$$
\begin{aligned}
& H_{\lambda-1}(1-z) f=-\tau(1-z) f, \\
& H_{\lambda-1}(1+z) f=\tau(1+z) f .
\end{aligned}
$$

Proof of assertion (a) of Theorem 4.11. With $N$ defined above, set $\mu=\lambda+$ $N$. Then by definition, $(1-z)^{-\mu} \in H^{p}$ and $(1-z)^{-\mu-1} \notin H^{p}$. The condition $p \geq 2$ implies that $\operatorname{Re} \mu \in(-1 / 2,1 / 2) \backslash\{0\}$. The role of $a^{\prime}$ is now played by $-\mu$ and we get that $(1-z)^{-\mu}$ is an eigenfunction of $H_{\lambda}$ with eigenvalue

$$
-\frac{\pi}{\sin (\pi(-\mu))}=\frac{\pi}{\sin (\pi \mu)} \text {. }
$$

Taking into account Lemma 4.9 and applying formula (4.16) $(N-n)$-times starting with $\mathcal{H}_{\mu}(1-z)^{-\mu}$ and then formula (4.17) $n$-times, $0 \leq n \leq N$, we obtain

$$
\mathcal{H}_{\mu-(N-n)-n}(1-z)^{-\mu+(N-n)}(1+z)^{n}=(-1)^{n} \frac{\pi}{\sin (\pi \mu)}(1-z)^{-\mu+(N-n)}(1+z)^{n} .
$$

The substitution $\mu=N+\lambda$ finally shows that

$\mathcal{H}_{\lambda}(1-z)^{-\lambda-n}(1+z)^{n}=(-1)^{-n} \frac{\pi}{\sin (\pi \lambda)}(1-z)^{-\lambda-n}(1+z)^{n}, \quad 0 \leq n \leq N$.

It is worth noting that, in view of Lemma 4.9, all eigenfunctions occurring in this process are contained in the kernels of $\Phi_{\mu-n}, 0 \leq n \leq N$, respectively. Therefore, assertion (a) is proven.

To prove assertion (b) we need the following lemma. 
Lemma 4.14. Let $\mu \neq 0$ and let $M_{\mu}:=\operatorname{ker}\left(\mathcal{H}_{\lambda}-\mu I\right)$ be a non-zero eigenspace such that $\Phi_{\lambda}(f)=0$ for all $f \in M_{\mu}$ with $\mathcal{H}_{\lambda}$ acting on $H^{p}, 2 \leq p<\infty$. Then

(a) $M_{\mu}$ is invariant under the action of $D_{1, \lambda}$.

(b) $M_{\mu}$ is spanned by a subset of the functions (4.12) and $\mu$ can only take on two values - viz. $\pm \Lambda$.

(c) The systems $\left\{f_{n}: 0 \leq n \leq N\right.$ and is even $\}$ and $\left\{f_{n}: 0<n \leq\right.$ $N$ and is odd $\}$ are bases for $M_{\Lambda}$ and $M_{-\Lambda}$, respectively.

Proof. (a): Let $f \in M_{\mu}$. Then $D_{1, \lambda} f \in H^{p}$. For, we mention that

$$
\left(D_{1, \lambda} f\right)(x)=-(1+x)(1-x) f^{\prime}(x)+\lambda f(x), \quad x \in[0,1) .
$$

As pointed out in [15], the function $x \mapsto(1-x) f^{\prime}(x)$ belongs to $L^{p}[0,1]$. Hence, the function $x \mapsto\left(D_{1, \lambda} f\right)(x)$ belongs to $L^{p}[0,1]$, too. Lemma 4.10 then indicates that $\mathcal{H}_{\lambda} D_{1, \lambda} f \in H^{p}$ and the equality

$$
\mathcal{H}_{\lambda} D_{1, \lambda} f=D_{1, \lambda} \mathcal{H}_{\lambda} f=\mu D_{1, \lambda} f
$$

(cf. Theorem 4.7) entails that $D_{1, \lambda} f \in H^{p}$ and $D_{1, \lambda} f \in \operatorname{ker}\left(\mathcal{H}_{\lambda}-\mu I\right)$ by (4.18).

(b): Let $l$ be the dimension of the finite-dimensional space $M_{\mu}$, cf. Corollary 4.13. Consider the eigenvalue problem

$$
D_{1, \lambda} f=\nu f, \quad f \in \operatorname{ker}\left(\mathcal{H}_{\lambda}-\mu I\right),
$$

where $\nu$ is not specified. Notice that we only have to look for solutions which are eigenfunctions of $\mathcal{H}_{\lambda}$, more precisely, belong to $\operatorname{ker}\left(\mathcal{H}_{\lambda}-\mu I\right)$. It is known that the functions belonging to $\operatorname{ker}\left(\mathcal{H}_{\lambda}-\mu I\right)$ must extend analytically in $\mathbb{C} \backslash[1, \infty)$. Using that $\operatorname{ker}\left(D_{1, \lambda}-v_{i} I\right)$ is spanned by

$$
f_{v_{i}}(z)=(1-z)^{\left(-\lambda+v_{i}\right) / 2}(1+z)^{\left(-\lambda-v_{i}\right) / 2}
$$

and has to belong to $\operatorname{ker}\left(\mathcal{H}_{\lambda}-\mu I\right)$, we get that $\left(-\lambda-v_{i}\right) / 2$ necessarily belongs to $\mathbb{Z}_{+}$. If $\left(-\lambda+v_{i}\right) / 2=n_{i} \in \mathbb{Z}_{+}$, then $\left(-\lambda+v_{i}\right) / 2=-\lambda-n_{i}$ and $(1-z)^{-\lambda-n_{i}} \in H^{p}$. Now let $N$ is the largest integer such that $(1-z)^{-\lambda-N} \in$ $H^{p}$. Choose an integer $n$ such that $0 \leq n \leq N$ and set

$$
f_{n}(z)=(1-z)^{-\lambda-n}(1+z)^{n} \text {. }
$$

Obviously,

$$
f_{n} \in \operatorname{ker}\left(D_{1, \lambda}-(-\lambda-2 n) I\right), \quad 0 \leq n \leq N,
$$

and this system coincides with (4.12). As a consequence we get that $\mu$ can take on only 2 values, namely $\pm \Lambda$. Let $\mathcal{V}_{e}$ and $\mathcal{V}_{o}$ be the systems defined in Theorem 4.11(b). Then $\mathcal{V}_{e}$ and $\mathcal{V}_{o}$ are systems of linearly independent vectors belonging to $M_{\Lambda}$ or $M_{-\Lambda}$, respectively. We claim that $\mathcal{V}_{e}$ and $\mathcal{V}_{o}$ are bases of these spaces, respectively. Let us denote the dimensions of spaces $M_{\Lambda}$ and $M_{-\Lambda}$ by $l_{e}$ and $l_{o}$, respectively. Then $l_{e}+l_{o}=l$. Suppose for instance that $\mathcal{V}_{e}$ is not a basis of $M_{\Lambda}$. Denote by $k_{l}$ the number of the elements in $\mathcal{V}_{e}$. Then $k_{e}<l_{e}$, and the characteristic polynomial of $D_{1, \lambda}: M_{\Lambda} \rightarrow M_{\Lambda}$ has degree larger than $k_{e}$. Thus there is a further eigenvector, say $\xi$ of $D_{1, \lambda}$ such that $\xi \neq 0$ and $\xi \notin \operatorname{span} \mathcal{V}_{e}$. Since, $\xi \in \operatorname{ker}\left(\mathcal{H}_{\lambda}-\Lambda I\right)$, the above considerations show that a scalar multiple of $\xi$ belongs to $\mathcal{V}_{e}$, and we get a contradiction. 
The claim (c) is obvious if one takes into account claim (a) of Theorem 4.11.

Assertion (b) of Theorem 4.11 is now a consequence of Lemma 4.14.

Proof of assertion (d) of Theorem 4.11. The condition

$$
-\frac{1}{2} \leq \operatorname{Re}(\lambda)+n-1<0
$$

ensures that $-\lambda-n$ belongs to the strip

$$
\{b \in \mathbb{C}:-1<\operatorname{Re}(b) \leq-1 / 2\} .
$$

Now set $a=-\lambda-n$ and $a^{\prime}=\lambda+n-1$. Clearly, there is a $\nu \in \mathbb{C}$ such that $a$ and $a^{\prime}$ are the solutions of the equation (4.7). Then it follows from (c) that

$$
g(z)=(1-z)^{-\lambda-n} F(-\lambda-n-1,-n ; \lambda ; z)
$$

is an eigenfunction of $\mathcal{H}_{\lambda}: H^{p} \rightarrow H^{p}$ with the eigenvalue

$$
\frac{-\pi}{\sin (\pi(-\lambda-n))}=(-1)^{n} \frac{\pi}{\sin (\pi \lambda)}
$$

if

$$
-\frac{1}{p}<\operatorname{Re}(-\lambda)-n
$$

To prove the last claim in (d), we have to show that the polynomial $F(-\lambda-$ $n+1,-n ; \lambda ; z)=F(-n,-\lambda-n+1 ; \lambda ; z)$ does not vanish at the point $z=1$. The proof is based on the following assertion from [14], formulated as a lemma here.

Lemma 4.15. (cf. [14, p. 277, Problem 4]). The hypergeometric polynomials $F(-k, \beta ; \gamma ; z)=F(\beta,-k ; \gamma ; z), \gamma \notin \mathbb{Z}_{-}$and $k \in \mathbb{Z}_{+}$can be defined as the expansion coefficients of the generating function

$$
w(z, t)=(1-t)^{\beta-\gamma}(1-t+z t)^{-\beta}=\sum_{k=0}^{\infty} \frac{(\gamma)_{k}}{k !} F(-k, \beta ; \gamma ; z) t^{k},
$$

where $|t|<1$ and $|t(1-z)|<1$.

We set $\gamma=\lambda$ and $\beta=-\lambda-n+1$. Consider

$$
w(1, t)=(1-t)^{-2 \lambda-n+1}=\sum_{k=0}^{\infty} \frac{(\gamma)_{k}}{k !} F(-k,-\lambda-n+1 ; \lambda ; 1) t^{k} .
$$

If $-2 \lambda-n+1 \notin \mathbb{Z}_{+}$, then all expansion coefficients are equal to $(-1)^{k}\left(\begin{array}{c}-2 \lambda-n+1 \\ k\end{array}\right)$, respectively. Thus we get $F(\lambda-n+1,-n ; \lambda ; 1) \neq 0$. Now, let $-2 \lambda-n+1 \in \mathbb{Z}_{+}$. Then $\operatorname{Im}(\lambda)=0$ and $-2 \lambda$ is an integer. Since $-1<-\lambda-n \leq-1 / 2$, we obtain $-2+2 n<-2 \lambda \leq-1+2 n$ and therefore, $-\lambda=n-1 / 2$. Consequently, $\beta-\gamma=2(n-1 / 2)-n+1=n$. This yields $F(\lambda-n+1,-n ; \lambda ; 1)=1$. Thus the proof of assertion (d) and the whole Theorem 4.11 is complete. 
Remark 4.16. The linear independence of the systems $\mathcal{U}_{e}$ and $\mathcal{U}_{o}$ can be established as follows. The functions

$$
g_{k}(z)=(1-z)^{-\lambda-k} F(-\lambda-k+1,-k ; \lambda ; z)
$$

can be written as

$$
g_{k}(z)=(1-z)^{-\lambda-N}(1-z)^{N-k} F(-\lambda-k+1,-k ; \lambda ; z) .
$$

It is easily seen that these systems are linearly independent if $F(-\lambda-k+$ $1,-k ; \lambda ; z) \neq 0$. This is almost obvious if in Lemma 4.15 one sets $\beta=-\lambda-$ $k+1$ and $\gamma=\lambda$.

If $-2 \lambda-k+1 \notin \mathbb{Z}_{+}$, then the expansion coefficients of $w(1, t)=(1-$ $t)^{-2 \lambda-k+1}$ are not equal to zero. If $-2 \lambda-k+1 \in \mathbb{Z}_{+}$, then $\operatorname{Im}(\lambda)=0$ and $-2 \lambda$ is an integer. If $2 \leq p<\infty$, then $(1-z)^{-\lambda-N} \in H^{p}$ and $(1-z)^{-\lambda-N-1} \notin H^{p}$. This yields that

$$
-\frac{1}{2}<-\lambda-N \leq \frac{1}{2}
$$

Therefore,

$$
2 N-1<-2 \lambda \leq 2 N+1 \text {. }
$$

Since $\lambda \notin \mathbb{Z}_{-}$it follows that $-\lambda=N+1 / 2$ and, consequently, $-2 \lambda-k+1=$ $2 N+2-k$. Hence, the $k$-th expansion coefficient of $w(1, t)=(1-t)^{2 N+2-k}$, $0 \leq k \leq N$ is not equal to zero.

The proof of Theorem 4.11 is complete if one takes into account the following two lemmas.

Lemma 4.17. (cf. Aleman et al. [1, Theorem 4.1]) If $\mu \in \mathbb{C} \backslash\{0\}$ and $f \in$ $\operatorname{ker}\left(\mathcal{H}_{\lambda}-\mu I\right)$ with $\Phi_{\lambda}(f) \neq 0$, then $\operatorname{dim} \operatorname{ker}\left(\mathcal{H}_{\lambda}-\mu I\right)=1, \mathcal{H}_{\lambda}: H^{p} \rightarrow H^{p}$, $1<p<2$, and $f$ is an eigenfunction of $D_{2, \lambda}$.

Lemma 4.18. Let $\mathcal{H}_{\lambda}: H^{p} \rightarrow H^{p}, \lambda \neq \mathbb{C} \backslash \mathbb{Z}$. Then ker $\mathcal{H}_{\lambda}=\{0\}$.

Proof. Obviously, it suffices to prove the claim for $1<p<2$. Suppose that a function $f \neq 0$ belongs to the space $H^{p}$ and $\mathcal{H}_{\lambda} f=0$. By the Eq. (4.3) in Theorem 4.7, we have $\mathcal{H}_{\lambda} D_{2, \lambda} f=D_{2, \lambda} \mathcal{H}_{\lambda} f=0$, and this implies that $f$ and $D_{2, \lambda} f$ both belong to ker $\mathcal{H}_{\lambda}$. By Theorem 4.5, part (II), we obtain that $\operatorname{dim} \operatorname{ker} \mathcal{H}_{\lambda}=1$. But this result implies that $D_{2, \lambda} f=\tau f$, where $\tau$ is a complex number. Theorem 4.11 entails that the solutions of the eigenvalue problem $D_{2, \lambda} f=\tau f, f$ is analytic in $D$, form a one-dimensional space spanned by $f_{a}=f_{a^{\prime}}$, the functions $f_{a}$ and $f_{a^{\prime}}$ given by (4.8), where $a$ and $a^{\prime}$ are the solutions of the Eq. (4.7) with $\nu$ replaced by $\tau$. The theory of the mentioned eigenvalue problem is explained in Theorem 4.8, and Theorem 4.11 together with its proof shows the importance of Theorem 4.8 in the study of the eigenvalue problem of $\mathcal{H}_{\lambda}-\omega I$ in the spaces $H^{p}$. Due to the restriction $1<p<2$, an inspection of Theorem 4.11 shows that $f \in \operatorname{ker}\left(\mathcal{H}_{\lambda}-\omega I\right)$ for an $\omega \neq 0$. But this contradicts $\mathcal{H}_{\lambda} f=0$.

So far, all results in this section are proved under the assumption that $\lambda \notin \mathbb{C} \backslash \mathbb{Z}$. In the next section we show that they are in force also for $\lambda \in \mathbb{N}$. 


\section{The Spectrum of $\mathcal{H}_{k}, k \in \mathbb{N}$}

Theorem 1.5 entails that in the case at hand $\mathcal{H}_{k}: H^{2} \rightarrow H^{2}$ is self-adjoint, has no point spectrum and its spectrum is exactly the interval $[0, \pi]$. Along with Theorem 4.1, this leads to the following results.

Corollary 5.1. Let $1<p<\infty$.

(a) The essential spectrum of $\mathcal{H}_{k}: H^{p} \rightarrow H^{p}$ coincides with the range of the function $\pi i \nu_{p}$.

(b) The operator $\beta I-\mathcal{H}_{k}: H^{p} \rightarrow H^{p}$ is invertible for $\beta \notin M_{p}$.

Corollary 5.2. If $1<p<2$, then the point spectrum of $\mathcal{H}_{k}$ coincides with $M_{p}^{0}$ and for $\beta \in M_{p}^{0}$ the operator $\beta I-\mathcal{H}_{k}$ is Fredholm with index 1 and $\operatorname{ker}\left(\beta I-\mathcal{H}_{k}\right)$ is the one-dimensional space spanned by

$$
(1-z)^{a} F(a+1, a+k ; \lambda ; z),
$$

where $a,-1 / p<\operatorname{Re}(a) \leq-1 / 2$, is uniquely defined by $-\pi /(\sin (\pi a))=\beta$, and $\beta I-\mathcal{H}_{k}$ is right-invertible.

Proof. Theorem 4.7(III) entails that all eigenvalues of $\mathcal{H}_{k}$ have positive real parts, and all eigenvalues have the multiplicity 1 . Since $k \in \mathbb{N}$, no eigenvalue $\pm \pi /(\sin (k \pi))$ exists. Further, $M_{p}^{0}$ is a part of the point spectrum of $\mathcal{H}_{k}$. Since $M_{p}^{0} \subset\{z \in \mathbb{C}: \operatorname{Re}(z)>0\}$, there is a uniquely defined complex number $a$ such that $-1 / p<\operatorname{Re}(a) \leq-1 / 2$ and

$$
\beta=-\frac{\pi}{\sin (\pi a)}=\frac{\pi}{\sin (\pi \mu)},
$$

where $\mu=a+1$ and therefore $1 / q<\operatorname{Re}(\mu) \leq 1 / 2$. Using Theorem 1.3 we see that the eigenspace with respect to the eigenvalue $\beta=-\pi /(\sin (\pi a))$ is spanned by

$$
X_{k, a}(z)=\sum_{n=0}^{\infty} x_{n}(k, a+1) z^{k},
$$

with $x_{n}(k, a+1)$ defined in the formulation of Theorem 1.3 (see also Assertion 1 in Remark 4.6).

Since $M_{p}^{0} \subset\{z \in \mathbb{C}: \operatorname{Re}(z)>0\}$, there is a uniquely defined complex number $a$ such that $-1 / p<\operatorname{Re}(a) \leq-1 / 2$ and

$$
\beta=-\frac{\pi}{\sin (\pi a)}=\frac{\pi}{\sin (\pi \mu)},
$$

where $\mu=a+1$. Clearly, $1 / q<\operatorname{Re}(\mu) \leq 1 / 2$. Using Theorem 1.3 , we get that $\beta$ is a latent root of $\mathcal{H}_{k}$ and the related root vectors form a one-dimensional space spanned by

$$
X_{k, a}(z):=\sum_{n=0}^{\infty} x_{n}(k, a+1) z^{n},
$$

where $x_{n}(k, a+1)$ is defined in formulation of Theorem 1.3. 
This claim is also true for any $\lambda \in\{z \in \mathbb{C}: \lambda \neq k$ and $|k-\lambda|<1 / 2\}$, $k \in \mathbb{N}$ fixed. But for these $\lambda$ we also have by Theorem 4.11 that the related eigenspaces are one-dimensional and are spanned by

$$
f_{a}(z)=(1-z)^{a} F(a+1, a+\lambda ; \lambda ; z),
$$

$-1 / p<\operatorname{Re}(a) \leq-1 / 2$ and $a$ is such that $-\pi /(\sin (\pi a))=\beta$. Notice that $M_{p}^{0}$ does not depend on $\lambda$, but the eigenfunctions do. We get, $k$ replaced by $\lambda$, that the functions (5.3) and (5.4) are connected by

$$
f_{a}(z)=\eta X_{\lambda, a}(z),
$$

where $\eta$ is a complex number. Since $f_{a}(0)=1$ and

$$
X_{\lambda, a}(0)=\frac{\Gamma(1+a) \Gamma(-a)}{\Gamma(\lambda)}
$$

we obtain that

$$
f_{a}(z)=\frac{\Gamma(\lambda)}{\Gamma(1+a) \Gamma(-a)} X_{\lambda, a}(z), \quad \lambda \neq k .
$$

Expanding $f_{a}(z)$ and $X_{\lambda, a}(z)$ in power series

$$
\begin{aligned}
& f_{a}(z)=\sum_{n=0}^{\infty} \xi_{n}(\lambda, a+1) z^{n}, \\
& X_{\lambda, a}(z)=\sum_{n=0}^{\infty} \frac{\Gamma(\lambda)}{\Gamma(1+a) \Gamma(-a)} x_{n}(\lambda, a+1),
\end{aligned}
$$

we get

$$
\xi_{n}(\lambda, a+1)=\frac{\Gamma(\lambda)}{\Gamma(1+a) \Gamma(-a)} x_{n}(\lambda, a+1),
$$

where

$$
\xi_{n}(\lambda, a+1)=\sum_{k=0}^{n} \eta_{k} \zeta_{n-k}
$$

Here, $\eta_{k}$ are the Taylor coefficients of $(1-z)^{a}$ and

$$
\zeta_{j}=\frac{(a+1)_{j}(a+\lambda)_{j}}{(\lambda)_{j} j !} .
$$

Theorem 4.1(c) indicates that in the case $\beta \notin W_{p}, 1<p<\infty$, the operator $\beta I-\mathcal{H}_{k}$ is invertible. Finally, if $\beta$ belongs to the essential spectrum of $\mathcal{H}_{k}$, then for $\beta=0$ it follows that ker $\mathcal{H}_{k}=\{0\}$ by Lemma 4.18, and if $\beta \in \operatorname{ess~sp} \mathcal{H}_{k}$, $\beta \neq 0$ then $\beta=-\pi /(\sin (\pi a))$ and $\operatorname{Re}(a)=1 / p$. If $\beta$ is an eigenvalue of $\mathcal{H}_{k}$, then the related eigenspace is spanned by $f_{a}(z)(1-z)^{a} F(a+1, a+k ; k ; z)$. Indeed, choose $p^{\prime}$ such that $1<p^{\prime}<p$. Then $\beta \in M_{p^{\prime}}^{0}$ and the claim follows. But $f_{a}(z) \notin H^{p}$ by Theorem $4.8(\mathrm{i})$. Thus the point spectrum of $\mathcal{H}_{\lambda}$ equals $M_{p}^{0}, 1<p<2$. 
Remark 5.3. The proof of Corollary 5.2 also yields that

$$
\xi_{n}(\lambda, a+1)=\frac{\Gamma(\lambda)}{\Gamma(1+a) \Gamma(-a)} x_{n}(\lambda, a+1)
$$

for all $a$ with $-1<\operatorname{Re}(a) \leq-1 / 2$ and all $\lambda \in \mathbb{C} \backslash \mathbb{Z}_{-}$.

Remark 5.4. In the case at hand, we identify the dual of $H^{p}$ with $H^{q}, 1 / p+$ $1 / q=1$, via the mapping $g \in H^{q} \mapsto\left(H^{p}\right)^{\prime}$, where

$$
<g, f>:=\frac{1}{2 \pi} \int_{0}^{2 \pi} g\left(e^{-i \theta} f\left(e^{i \theta}\right) d \theta .\right.
$$

Then the dual or transpose for the operator $\mathcal{H}_{\lambda}: H^{p} \rightarrow H^{p}$ is the operator $\mathcal{H}_{\lambda}: H^{q} \rightarrow H^{q}$. For the concept of the transpose operator and its connection with Fredholm theory see [13, Chapters 15, 17].

Corollary 5.5. If $2<p<\infty$, then $\mathcal{H}_{k}: H^{p} \rightarrow H^{p}$ has no point spectrum and for $\beta \in M_{p}^{0}$, the operator $\beta I-\mathcal{H}_{k}: H^{p} \rightarrow H^{p}$ is Fredholm with index -1 . Moreover, this operator is left-invertible.

Proof. The claim follows from Corollary 5.2 by duality.

Corollary 5.6. For all $k \in \mathbb{N}$ the kernel of $\mathcal{H}_{k}$ is trivial.

Indeed, the result follows from Theorem 4.5(III).

\section{The Main Theorem}

This section is aimed at the formulation of the Main Theorem, which is a consequence of the results pointed out in Sections 3-5.

Theorem 6.1. (Main Theorem). Let $\lambda \in \mathbb{C} \backslash \mathbb{Z}_{-}$and $\mathcal{H}_{\lambda}: H^{p} \rightarrow H^{p}, 1<p<$ $\infty$.

(I) The essential spectrum of $\mathcal{H}_{\lambda}$ is equal to the image of the function $\pi i \nu_{p}$. Moreover, if $\beta \in W_{p}^{0}$, then $\beta I-\mathcal{H}_{\lambda}$ is Fredholm and

$$
\begin{aligned}
& \operatorname{ind}_{H^{p}}\left(\beta I-\mathcal{H}_{\lambda}\right)=1, \quad 1<p<2, \\
& \operatorname{ind}_{H^{p}}\left(\beta I-\mathcal{H}_{\lambda}\right)=-1, \quad 2<p<\infty .
\end{aligned}
$$

If $\beta \notin W_{p}, 1<p<\infty$, then the operator $\beta I-\mathcal{H}_{\lambda}$ acting on $H^{p}$ is Fredholm with index zero. If $\lambda$ is real and $\lambda>1 / 2$, then $\beta I-\mathcal{H}_{\lambda}$ is invertible on $H^{p}$.

(II) The point spectrum of $\mathcal{H}_{\lambda}$ is described by the following assertions.

(a) Let $p \geq 2$ and $(1-z)^{-\lambda} \in H^{p}$, i.e. $\operatorname{Re}(\lambda)<1 / p$. If $N \geq 0$ is the largest integer such that $(1-z)^{-\lambda-N} \in H^{p}$, then

$$
f_{n}(z):=(1-z)^{-n-\lambda}(1+z)^{n}, \quad 0 \leq n \leq N,
$$

are eigenfunctions of $\mathcal{H}_{\lambda}$ related to the eigenvalue

$$
(-1)^{n} \frac{\pi}{\sin (\lambda \pi)}
$$


(b) For any $p \geq 2$ set

$$
\begin{aligned}
& \mathcal{V}_{e}:=\left\{f_{n} \in H^{p}: 0 \leq n \leq N, n \text { is even }\right\}, \\
& \mathcal{V}_{o}:=\left\{f_{n} \in H^{p}: 0<n \leq N, n \text { is odd }\right\} .
\end{aligned}
$$

Then the systems $\mathcal{V}_{e}$ and $\mathcal{V}_{o}$ are bases of the kernels of $\mathcal{H}_{\lambda}-$ $(\pi / \sin (\pi \lambda)) I$ and $\mathcal{H}_{\lambda}+(\pi / \sin (\pi \lambda)) I$, respectively. Moreover, every eigenfunction of $\mathcal{H}_{\lambda}$ belongs to $\operatorname{ker}\left(\mathcal{H}_{\lambda}-(\pi / \sin (\pi \lambda)) I\right)$ or to $\operatorname{ker}\left(\mathcal{H}_{\lambda}+(\pi / \sin (\pi \lambda)) I\right)$.

(c) If $p<2$, then the point spectrum of $\mathcal{H}_{\lambda}$ is the image of the set

$$
\left\{-\frac{1}{p}<\operatorname{Re}(a) \leq-\frac{1}{2}\right\} \cup \Omega_{\lambda}
$$

under the map $a \mapsto-\pi / \sin (\pi a)$ or, equivalently, under the map $a \mapsto-\pi i / \sinh (-\pi i a)$, where

$\Omega_{\lambda}:=\left\{\xi \in\{-\lambda,-\lambda-1\}:-\frac{\pi}{\sin (\pi \xi)}\right.$ is in the point spectrum of $\left.\mathcal{H}_{\lambda}: l^{2} \rightarrow l^{2}\right\}$.

Each eigenvalue $-\pi / \sin (\pi a), a \neq-\lambda,-\lambda-1$ has multiplicity one and the corresponding eigenspace is spanned by

$$
f_{a}(z):=(1-z)^{a} F(a+1, a+\lambda ; \lambda ; z) .
$$

(d) Let $n \in \mathbb{Z}_{+}$be such that $-1 / 2 \leq \operatorname{Re}(\lambda)+n-1<0$. Then

$-1<\operatorname{Re}(-\lambda)-n \leq-1 / 2, \quad 1 / 2-n \leq \operatorname{Re}(\lambda)<1-n$.

If $-1 / p<\operatorname{Re}(-\lambda)-n$, then $\mathcal{H}_{\lambda}: H^{p} \rightarrow H^{p}$ owns an eigenfunction, viz.

$$
g(z):=(1-z)^{-\lambda-n} F(-\lambda-n+1,-n ; \lambda ; z)
$$

with the eigenvalue

$$
(-1)^{n} \frac{\pi}{\sin (\pi \lambda)}
$$

Moreover, $g \notin H^{2}$.

(III) Let $1<p<\infty$. If $\beta \notin M_{p}$ and $\beta \neq \pm \pi /(\sin (\pi \lambda))$, $\lambda \notin \mathbb{N}$, then $\beta I-\mathcal{H}_{\lambda}$ is invertible.

Remark 6.2. Assertion (d) shows that $\mathcal{H}_{\lambda}$ may possess a one-dimensional eigenspace with eigenvalue $\pi /(\sin (\pi \lambda))$ or $-\pi /(\sin (\pi \lambda))$, which is not contained in $H^{2}$. It is not known whether there exists a higher-dimensional eigenspace with the properties mentioned.

\section{The spectrum of $\mathcal{H}_{\lambda}: l^{p} \rightarrow l^{p}, 1<p<\infty$.}

We now show that the results of the previous considerations can be used in order to describe the spectrum of $\mathcal{H}_{\lambda}: l^{p} \rightarrow l^{p}, 1<p<\infty$. An important tool to achieve this aim is the celebrated Hausdorff-Young Theorem. Recall that $\mathcal{F}$ denotes the Fourier transform defined on $L^{p}, 1 \leq p \leq \infty$, i.e. it is the map that sends an element $f \in L^{p}$ into the sequence $\left(\widehat{f}_{n}\right)_{n \in \mathbb{Z}}$ of its Fourier coefficients. It is well-known that $\mathcal{F}$ is one-to-one. 
It is important to underline that in this section the space $H^{p}$ is identified with the image im $P$ of the Riesz projection $P$. Equivalently, $H^{p}$ is identified with the space of all boundary functions. All eigenfunctions of $\mathcal{H}_{\lambda}$ described in Theorem 6.1 can be analytically extended into $\mathbb{C} \backslash[1, \infty)$. For this continuations we will keep the notations used in Theorem 6.1. Hence, if $f$ is an eigenfunction of $\mathcal{H}_{\lambda}$, then $f(t)$ is defined for all $t \in \mathbb{T} \backslash\{1\}$. The latter function is the boundary function of $z \mapsto f(z),|z|<1$. The shift operator in $H^{2}=\operatorname{im} P$ is now defined as $h \mapsto \chi_{1} h, \chi_{1}(t)=t$, and is again denoted by $V$. It should not cause a misunderstanding that in both cases we denote the shift in $H^{p}$ and im $P$ by $V$ because it is clear from the context where the corresponding shift acts.

Theorem 7.1. (Hausdorff-Young Theorem, cf. [5, Section 13.5]). Let $1 / p+$ $1 / q=1$ if $q \in(1, \infty)$ and $p=\infty$ if $q=1$.

1. If $g \in H^{q}, 1 \leq q \leq 2$, then $\mathcal{F} g \in l^{p}$ and $\|\mathcal{F} g\|_{l^{p}} \leq\|g\|_{H^{q}}$.

2. If $\varphi \in l^{q}, 1 \leq q \leq 2$, then there is an element $g \in H^{p}$ such that $\varphi=\mathcal{F} g$ and $\|g\|_{H^{p}} \leq\|g\|_{l^{q}}$.

In what follows we will write $\left.\mathcal{H}_{\lambda}\right|_{H^{p}}$ and $\left.\mathcal{H}_{\lambda}\right|_{l^{p}}$ instead of $\mathcal{H}_{\lambda}: H^{p} \rightarrow H^{p}$ and $\mathcal{H}_{\lambda}: l^{p} \rightarrow l^{p}$, respectively.

Recall that by Theorem $4.1(\mathrm{a}),(\mathrm{b})$, the operators $\left.\left(\beta I-\mathcal{H}_{\lambda}\right)\right|_{H^{p}}$ and $\left.\left(\beta I-\mathcal{H}_{\lambda}\right)\right|_{l^{q}}, 1 / p+1 / q=1, p \in(1, \infty)$ are simultaneously Fredholm with the same Fredholm indices.

Theorem 7.2. Let $p \in(1, \infty)$ and $1 / p+1 / q=1$. If $\left.\left(\beta I-\mathcal{H}_{\lambda}\right)\right|_{H^{q}}$ is Fredholm, then the Fourier transform is an isomorphism between the spaces $\operatorname{ker}(\beta I-$ $\left.\mathcal{H}_{\lambda}\right)\left.\right|_{H^{q}}$ and $\left.\operatorname{ker}\left(\beta I-\mathcal{H}_{\lambda}\right)\right|_{l^{p}}$. In particular, an element $\varphi \in l^{p}$ is in $\operatorname{ker}(\beta I-$ $\left.\mathcal{H}_{\lambda}\right)\left.\right|_{l^{p}}$ if and only if $\varphi=\mathcal{F} g$ for a function $\left.g \in \operatorname{ker}\left(\beta I-\mathcal{H}_{\lambda}\right)\right|_{H^{q}}$.

A much more general result is proved in [3]. For the convenience of the reader, we give the proof of Theorem 7.2 based on the ideas of [3].

Proof. Let $\widehat{H}^{p}, p \in(1, \infty)$ be the space of all sequences $\left(g_{k}\right)_{k \in \mathbb{Z}_{+}}$for which there is a function $g \in H^{p}$ such that $\mathcal{F} g=\left(g_{k}\right)_{k \in \mathbb{Z}_{+}}$. Let us equip $\widehat{H}^{p}$ with the norm

$$
\left\|\left(g_{k}\right)_{k \in \mathbb{Z}_{+}}\right\|:=\|g\|_{H^{p}} .
$$

Apparently the spaces $\widehat{H}^{p}$ and $H^{p}$ are isometrically isomorphic and the operator $\left.\mathcal{H}_{\lambda}\right|_{H^{p}}, \lambda \notin \mathbb{Z}_{-}$induces a linear bounded operator on $\widehat{H}^{p}$ by its matrix representation

$$
\left[\left.\mathcal{H}_{\lambda}\right|_{H^{p}}\right]:=\left(\frac{1}{n+m+\lambda}\right)_{n, m=0}^{\infty}
$$

with respect to the Schauder basis $\left\{\chi_{n}\right\}_{n \in \mathbb{Z}_{+}}$. Assume that $2 \leq p<\infty$. The first part of Hausdorff-Young Theorem shows that $\widehat{H}^{q}$ is continuously embedded in the space $l^{p}$. Moreover, $\widehat{H}^{q}$ is dense in $l^{p}$. The operators $(\beta I-$ $\left.\mathcal{H}_{\lambda}\right)\left.\right|_{H^{q}}$ and $\left[\left.\left(\beta I-\mathcal{H}_{\lambda}\right)\right|_{H^{q}}\right]$ acting, respectively, on $H^{q}$ and $\widehat{H}^{q}$ have the same Fredholm properties as well as $\left.\left(\beta I-\mathcal{H}_{\lambda}\right)\right|_{l^{p}}$. The operator $\left.\left(\beta I-\mathcal{H}_{\lambda}\right)\right|_{l^{p}}$ 
is an extension of the operator $\left[\left.\left(\beta I-\mathcal{H}_{\lambda}\right)\right|_{H^{q}}\right]$ on the whole space $l^{p}$. By Lemma 2.1,

$$
\left.\operatorname{ker}\left(\beta I-\mathcal{H}_{\lambda}\right)\right|_{l^{p}}=\operatorname{ker}\left[\left.\left(\beta I-\mathcal{H}_{\lambda}\right)\right|_{H^{q}}\right] .
$$

whence the assertion of Theorem 7.2 follows for $p \geq 2$.

Now let $1<p<2$. The second part of Hausdorff-Young Theorem assures that $l^{p}$ is continuously embedded into $\widehat{H}^{q}$. Clearly, $\left[\left.\left(\beta I-\mathcal{H}_{\lambda}\right)\right|_{H^{q}}\right]$ is an extension of $\left.\left(\beta I-\mathcal{H}_{\lambda}\right)\right|_{l^{p}}$. Using Lemma 2.1 once more, we obtain the result for $1<p<2$.

Remark 7.3. The proof of Theorem 7.2 indicates that

(a) $\mathcal{F}\left\{\left.\left(\beta I-\mathcal{H}_{\lambda}\right)\right|_{H^{q}}(g)\right\}=\left[\left.\left(\beta I-\mathcal{H}_{\lambda}\right)\right|_{H^{p}}\right](\mathcal{F} g)=\left.\left(\beta I-\mathcal{H}_{\lambda}\right)\right|_{l^{p}}(\mathcal{F} g)$ for $g \in H^{q}, 1<q \leq 2$ and

(b) $\left.\left(\beta I-\mathcal{H}_{\lambda}\right)\right|_{l^{q}}(\varphi)=\left[\left.\left(\beta I-\mathcal{H}_{\lambda}\right)\right|_{H^{p}}\right](\mathcal{F} g)=\mathcal{F}\left\{\left.\left(\beta I-\mathcal{H}_{\lambda}\right)\right|_{H^{p}}(g)\right\}$, where $1<q<2, \varphi \in l^{q}$ and $g \in H^{p}$ is such that $\varphi=\mathcal{F} g$.

Corollary 7.4. Let $1<p<\infty$. Then $\left.\operatorname{ker} \mathcal{H}_{\lambda}\right|_{l^{p}}=\{0\}$.

Proof. By Lemma 4.18, we have ker $\left.\mathcal{H}_{\lambda}\right|_{l^{2}}=\{0\}$. Hence ker $\left.\mathcal{H}_{\lambda}\right|_{l^{p}}=\{0\}$ for $1<p<2$. For $1<p<\infty$ the claim can be proved using the fact that if $h:=\left(h_{n}\right)_{n \in \mathbb{Z}_{+}}$belongs to ker $\left.\mathcal{H}_{\lambda}\right|_{l^{p}}$, then the sequence

$$
g:=\left(\frac{h_{n}}{(n+\lambda)(n+\lambda+1)}\right)_{n \in \mathbb{Z}_{+}}
$$

belongs to ker $\left.\mathcal{H}_{\lambda+2}\right|_{l^{2}}$, cf. [7, Section3]. Since ker $\left.\mathcal{H}_{\lambda+2}\right|_{l^{2}}=\{0\}$, we obtain that $g$ and $h$ are equal to the zero sequence and we are done.

Corollary 7.5. Let $a \in\{b \in \mathbb{C}:-1<\operatorname{Re}(b)<-1 / 2\}$ and let $\lambda \in \mathbb{C} \backslash \mathbb{Z}_{-}$ be such that $\lambda-a-1 \notin \mathbb{Z}_{-}$. Then the expansion coefficients $\xi_{n}(\lambda, a+1)$ of $(1-z)^{a} F(a+1, a+\lambda ; \lambda ; z)$ own the asymptotic estimate

$$
\xi_{n}(\lambda, a+1) \sim \frac{\Gamma(\lambda) \Gamma(-2 a-1)}{\Gamma(-a)^{2} \Gamma(\lambda-a-1)} n^{-1-a} .
$$

Proof. The estimate (7.1) is an immediate consequence of [9, Section 6]. Of course one has to take Remark 5.3 into account.

Let $V: l^{p} \rightarrow l^{p}, 1<p<\infty$ be the operator defined by $\left(a_{0}, a_{1}, \ldots\right) \mapsto$ $\left(0, a_{0}, a_{1}, \ldots\right)$. This operator plays an important role in what follows.

Theorem 7.6. (Main Theorem for $l^{p}$-case). Let $\lambda \in \mathbb{C} \backslash \mathbb{Z}_{-}$and $\mathcal{H}_{\lambda}: l^{p} \rightarrow l^{p}$, $1<p<\infty$.

(I) The essential spectrum of $\mathcal{H}_{\lambda}$ is equal to the image of the function $\pi i \nu_{q}$. Moreover, if $\beta \in W_{q}^{0}$, then $\beta I-\mathcal{H}_{\lambda}$ is Fredholm and

$$
\begin{aligned}
& \operatorname{ind}_{l^{p}}\left(\beta I-\mathcal{H}_{\lambda}\right)=1, \quad 2<p<\infty, \\
& \operatorname{ind}_{l^{p}}\left(\beta I-\mathcal{H}_{\lambda}\right)=-1, \quad 1<p<2 .
\end{aligned}
$$

If $\beta \notin W_{q}^{0}$, then the operator $\beta I-\mathcal{H}_{\lambda}$ acting on $l^{p}, 1<p<\infty$ is Fredholm with index zero. If $\lambda$ is real and $\lambda>1 / 2$, then $\beta I-\mathcal{H}_{\lambda}$ is invertible on $l^{p}$. 
(II) The point spectrum of $\mathcal{H}_{\lambda}$ is described by the following assertions.

(a) Let $2 \leq q, 1 / p+1 / q=1$, and let $f_{n}, 0 \leq n \leq N$ be the functions given in (4.12). Then the function $\mathcal{F} f_{n}$ are eigenfunctions of $\left.\mathcal{H}_{\lambda}\right|_{l^{q}}$ related to the eigenvalues $(-1)^{n} \pi /(\sin (\pi \lambda))$.

(b) For any $q \leq 2$ set

$$
\mathcal{F} \mathcal{V}_{e}:=\left\{\mathcal{F} f_{n}: f_{n} \in \mathcal{V}_{e}\right\}
$$

$\mathcal{F} \mathcal{V}_{o}:=\left\{\mathcal{F} f_{n}: f_{n} \in \mathcal{V}_{o}\right\}$

Then every eigenfunction of $\left.\mathcal{H}_{\lambda}\right|_{l^{q}}$ belongs to $\left.\operatorname{ker}\left(\mathcal{H}_{\lambda}-\frac{\pi}{\sin (\pi \lambda)} I\right)\right|_{l^{q}}$ or to $\left.\operatorname{ker}\left(\mathcal{H}_{\lambda}+\frac{\pi}{\sin (\pi \lambda)} I\right)\right|_{l^{q}}$ and $\mathcal{F} \mathcal{V}_{e}$ and $\mathcal{F} \mathcal{V}_{o}$ are bases of these kernels, respectively.

(c) If $p>2$, then the point spectrum of $\left.\mathcal{H}_{\lambda}\right|_{l^{p}}$ is the image of the set

$$
\left\{-\frac{1}{q}<\operatorname{Re}(a) \leq-\frac{1}{2}\right\} \cup \Omega_{\lambda}
$$

under the map $a \mapsto \frac{-\pi}{\sin (\pi \lambda)}$ or, equivalently, under the map $a \mapsto$ $\frac{\pi}{\sinh (-\pi i a)}$, where

$\Omega_{\lambda}:=\left\{\xi \in\{-\lambda,-\lambda-1\}: \frac{-\pi}{\sin (\pi \xi)}\right.$ is in the point spectrum of $\left.\left.\mathcal{H}_{\lambda}\right|_{l^{2}}\right\}$.

Each eigenvalue $-\pi /(\sin (\pi a)), a \neq-\lambda,-\lambda-1$ has multiplicity one and the corresponding eigenspace is spanned by $\mathcal{F} f_{a}$, where

$$
f_{a}(z)=(1-z)^{a} F(a+1, a+\lambda ; \lambda ; z) .
$$

(d) Let $n \in \mathbb{Z}_{+}$be such that $-1 / 2 \leq \operatorname{Re}(\lambda)+n-1<0$. If $-1 / q<$ $\operatorname{Re}(-\lambda)-n$, then $\left.\mathcal{H}_{\lambda}\right|_{l^{p}}$ owns an eigenfunction, viz. $\mathcal{F}(g)$,

$$
g(z)=(1-z)^{-\lambda-n} F(-\lambda-n+1,-n ; \lambda ; z)
$$

with the eigenvalue

$$
(-1)^{n} \frac{\pi}{\sin (\pi \lambda)}
$$

Moreover, $g \notin l^{2}$.

(III) Let $1<p<\infty$. If $\beta \notin M_{p}$ and $\beta \neq \pm \pi /(\sin (\pi \lambda)), \lambda \notin \mathbb{N}$, then $\beta I-\mathcal{H}_{\lambda}$ is invertible.

Sketch of the proof. Claim I follows immediately from Theorem 4.1.

Claims II(a) and II(b): The boundary functions of the functions $f_{n}$, $0 \leq n \leq N$, given by (4.12) can be written as

$$
f(t)=(1-t)^{-N-\lambda}(1-t)^{N-n}(1+t)^{n}, \quad t \in \mathbb{T} \backslash\{1\} .
$$

It is well-known that the Fourier coefficients of the function $t \mapsto(1-t)^{-\gamma}$, $\gamma=N+\lambda, t \in \mathbb{T} \backslash\{1\}$, are given by $(-1)^{n}\left(\begin{array}{c}-\gamma \\ n\end{array}\right)$ for $n \in \mathbb{Z}_{+}$and by 0 for $n \in \mathbb{Z}_{-} \backslash\{0\}$. In order to show that the sequence $\left\{(-1)^{n}\left(\begin{array}{c}-\gamma \\ n\end{array}\right)\right\}, n \in \mathbb{Z}_{+}$, belongs to $l^{q}$ for $(1-t)^{-\gamma} \in H^{p}, p \geq 2$, we use the well-known estimate

$$
c^{-1}(n+1)^{-1+\operatorname{Re}(\gamma)} \leq\left|\left(\begin{array}{c}
-\gamma \\
n
\end{array}\right)\right| \leq c(n+1)^{-1+\operatorname{Re}(\gamma)},
$$


where $c$ is a constant not depending on $n$ (see, e.g. [2, Sections 6,19 and 6.21]). The condition $(1-t)^{-\gamma} \in H^{p}, t \in \mathbb{T} \backslash\{1\}$ implies $\operatorname{Re}(\gamma)<1 / p=1-1 / q$ and therefore $-1+1 / q<-\operatorname{Re}(\gamma)$. Hence, $1 / q<1-\operatorname{Re}(\gamma)$ and the claim is proved. Since

$$
f_{n}(t)=(I-V)^{N-n}(I+V)^{n}(1-t)^{-\gamma}, \quad t \in \mathbb{T} \backslash\{1\}
$$

belongs to $H^{p}, p \geq 2$ and

$$
\begin{aligned}
\mathcal{F} f_{n} & =\mathcal{F}\left\{(I-V)^{N-n}(I+V)^{n}(1-t)^{-\gamma}\right\} \\
& =(I-V)^{N-n}(I+V)^{n} \mathcal{F}\left((1-t)^{-\gamma}\right) \in l^{q},
\end{aligned}
$$

we get by Remark 7.3(b) that

$$
\begin{aligned}
& \left.\mathcal{F} f_{n} \in \operatorname{ker}\left(\mathcal{H}_{\lambda}-\frac{\pi}{\sin (\pi \lambda)}\right)\right|_{l^{q}} \text { if } n \text { is even, } \\
& \left.\mathcal{F} f_{n} \in \operatorname{ker}\left(\mathcal{H}_{\lambda}+\frac{\pi}{\sin (\pi \lambda)}\right)\right|_{l^{q}} \text { if } n \text { is odd. }
\end{aligned}
$$

Conversely, if $\left.h \in \operatorname{ker}\left(\mathcal{H}_{\lambda}-\xi I\right)\right|_{l^{q}}, \xi \neq 0$ and $1 \leq q \leq 2$, then Remark 7.3(b) entails that there is a function $f \in H^{p}$ such that $\mathcal{F} f=h$ and $f \in \operatorname{ker}\left(\mathcal{H}_{\lambda}-\right.$ $\xi I)\left.\right|_{H^{p}}, p \geq 2$. Since $p \geq 2$, by Theorem $4.11(\mathrm{~b})$ we have $\xi= \pm \pi /(\sin (\pi \lambda))$. Now it should be clear that (a) and (b) in Claim II are in force.

Claim II(c) is a consequence of Theorem 7.2 and Corollary 7.5. The relation (7.1) ensures in case $a=-1 / q+i \xi$ and $\lambda-a-1 \notin \mathbb{Z}_{-}$that the sequence $\mathcal{F}\left((1-t)^{-a} F(a+1, a+\lambda ; \lambda, t)\right)$ does not belong to $l^{p}$.

Claim II(d) also follows from Theorem 7.2.

\section{Outlook}

There are a variety of spaces such as Korenblum spaces, Bergman spaces, weighted Hardy and $l^{p}$-spaces, where the matrix operator $\mathcal{H}_{\lambda}$ is well-defined, at least for $\lambda=1$. So one may force the problem of describing the spectrum of the related Hilbert operator. The point spectrum of $\mathcal{H}_{\lambda}$ defined on the Korenblum spaces is studied in [1] but more is not known. Concerning Bergman spaces $A^{p}, 1<p<\infty$, it is only known that $\mathcal{H}_{1}$ is bounded on $A^{p}$ for $p>2$ but it does not act on $A^{p}, 1<p \leq 2$. So it becomes clear that the description of the spectrum of $\mathcal{H}_{\lambda}$ in these spaces is a challenge. Nevertheless, for weighted Hardy spaces and for $l^{p}$-spaces, the problem may be treatable, since there is a well-developed Fredholm theory for Toeplitz and Hankel operators acting in these spaces.

\section{Acknowledgements}

I would like to thank my colleague Victor Didenko for his valuable help and comments during the work on this paper.

Funding Information Open Access funding enabled and organized by Projekt DEAL. 
Open Access. This article is licensed under a Creative Commons Attribution 4.0 International License, which permits use, sharing, adaptation, distribution and reproduction in any medium or format, as long as you give appropriate credit to the original author(s) and the source, provide a link to the Creative Commons licence, and indicate if changes were made. The images or other third party material in this article are included in the article's Creative Commons licence, unless indicated otherwise in a credit line to the material. If material is not included in the article's Creative Commons licence and your intended use is not permitted by statutory regulation or exceeds the permitted use, you will need to obtain permission directly from the copyright holder. To view a copy of this licence, visit http:// creativecommons.org/licenses/by/4.0/.

Publisher's Note Springer Nature remains neutral with regard to jurisdictional claims in published maps and institutional affiliations.

\section{References}

[1] Aleman, A., Montes-Rodriguez, A., Sarafoleanu, A.: The eigenfunctions of the Hilbert matrix. Constr. Approx. 36, 353-372 (2012)

[2] Böttcher, A., Silbermann, B.: Analysis of Toeplitz Operators. Springer Monographs in Mathematics, 2nd edn. Springer, Berlin (2006)

[3] Didenko, V.D., Silbermann, B.: Kernels of a class of Toeplitz plus Hankel operators with piecewise continuous generating functions. In: Dick, J., Kuo, F.Y., Woźniakowski, H. (eds.) Contemporary Computational Mathematics: A Celebration of the 80th Birthday of Ian Sloan, pp. 317-337. Springer, Cham (2018)

[4] Dostanic, M., Jevtic, M., Vukotic, D.: Norm of the Hilbert matrix on Bergman and Hardy spaces and a theorem of Nehari type. J. Funct. Anal. 254, 2800-2815 (2008)

[5] Edwards, R.: Fourier Series. A Modern Introduction. Vol. 2. Graduate Texts in Mathematics, vol. 85. New York/Heidelberg/Berlin, Springer-Verlag (1982)

[6] Garnet, J. B.: Bounded Analytic Functions. In: Pure and Applied Mathematics, vol. 96. Academic Press, New York (1981)

[7] Gohberg, I., Krupnik, N.: Singular integral operators with piecewise continuous coefficients and their symbols. Math. USSR Izvestiya 5, 955-979 (1971)

[8] Fink, T., Roch, S., Silbermann, B.: Banach algebra generated by two idempotents and one flip. Math. Nachrichten 216, 73-94 (2000)

[9] Hill, C.K.: On the singly generated Hilbert matrix. J. London Math. Soc. 35, 17-29 (1960)

[10] Hagen, R., Roch, S., Silbermann, B.: Spectral Theory of Approximation Methods for Convolution Equations. In: Operator Theory: Advances and Applications, vol. 74. Birkhäuser Verlag, Basel (1995)

[11] Hollenbeck, B., Verbitzky, I.: Best constants for the Riesz projection. J. Funct. Anal. 175, 370-392 (2000)

[12] Koosis, P.: Introduction to $H^{p}$-Spaces, 2-nd edn. In: Cambridge Tracts in Mathematics, vol. 115. Cambridge University Press, Cambridge (1998)

[13] Lax, P.D.: Functional Analysis. Wiley-Interscience, New York (2020)

[14] Lebedev, N.N.: Special Functions and Their Applications. Dover Publications, New York (1972) 
[15] Lueking, D.H.: Forward and reverse Carleson inequalities for functions in Bergman spaces and their derivatives. Am. J. Math. 107, 85-111 (1985)

[16] Magnus, W.: On the spectrum of Hilbert matrix. Am. J. Math. 72, 699-704 (1950)

[17] Roch, S.: Local Algebras of Toeplitz operators. Mathem. Nachrichten 152, 69$81(1991)$

[18] Rosenblum, M.: On the Hilbert matrix I. Proc. Am. Math. Soc. 9, 135-140 (1958)

[19] Rosenblum, M.: On the Hilbert matrix II. Proc. Am. Math. Soc. 9, 581-584 (1958)

[20] Roch, S., Santos, P. A., Silbermann, B.: Non-Commutative Gelfand Theories. A Tool-Kit for Operator Theorists and Numerical Analysts. Universitext. Springer-Verlag London Ltd., London (2011)

[21] Roch, S., Silbermann, B.: Algebras of Convolution Operators and Their Image in the CAlkin Algebra. Report MATH 90. Akademie der Wissenschaften der DDR Karl-Weierstrass-Institut für Mathematik, Berlin (1990)

[22] Roch, S., Silbermann, B.: A handy formula for the Fredholm index of Toeplitz plus Hankel operators. Indag. Math. 23(4), 663-689 (2012)

[23] Siskakis, A.G.: The Hilbert matrix operator on spaces of analytic functions. Internet, Madrid (2012)

Bernd Silbermann $(\bowtie)$

Technische Universität Chemnitz

Fakultät für Mathematik

09107 Chemnitz

Germany

e-mail: silbermn@mathematik.tu-chemnitz.de

Received: December 21, 2020.

Accepted: April 8, 2021. 